\title{
Measuring residual strain and stress in thermal spray coatings using neutron diffractometers.
}

FAISAL, N.H., AHMED, R., PRATHURU, A.K., PARADOWSKA, A. and LEE, T.L. 


\title{
Measuring Residual Strain and Stress in Thermal Spray Coatings Using Neutron Diffractometers
}

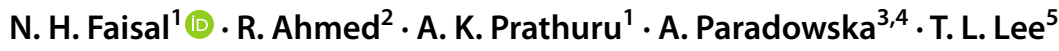

Received: 29 March 2021 / Accepted: 25 October 2021 / Published online: 10 November 2021

(c) The Author(s) 2021

\begin{abstract}
Background During thermal spray coating, residual strain is formed within the coating and substrates due to thermomechanical processes and microstructural phase changes.

Objective This paper provides a comprehensive guide to researchers planning to use neutron diffraction technique for thermal spray coatings, and reviews some of these studies.

Methods ENGIN-X at the ISIS spallation source is a neutron diffractometer (time-of-flight) dedicated to materials science and engineering with high resolution testing. The focus is on the procedure of using ENGIN-X diffractometer for thermal spray coatings with a view that it can potentially be translated to other diffractometers.

Results Number of studies involving neutron diffraction analysis in thermal spray coatings remain limited, partly due to limited number of such strain measurement facilities globally, and partly due to difficulty is applying neutron diffraction analysis to measure residual strain in the thermal spray coating microstructure.

Conclusions This technique can provide a non-destructive through-thickness residual strain analysis in thermally sprayed components with a level of detail not normally achievable by other techniques. Neutron sources have been used to measure strains in thermal spray coatings, and here, we present examples where such coatings have been characterised at various neutron sources worldwide, to study residual strains and microstructures.
\end{abstract}

Keywords Residual strain $\cdot$ Residual stress $\cdot$ Neutron diffraction techniques $\cdot$ Thermal spray coatings $\cdot$ ENGIN-X $\cdot$ Neutron diffractometers

\section{Introduction}

This paper presents test methodologies for experimentally determining residual strain (and stress) in thermal spray coatings using neutron diffraction technique with emphasis

\section{N. H. Faisal \\ N.H.Faisal@rgu.ac.uk}

1 School of Engineering, Robert Gordon University, Garthdee Road, Aberdeen AB10 7GJ, UK

2 School of Engineering and Physical Sciences, Heriot-Watt University, Edinburgh EH14 4AS, UK

3 Australian Nuclear Science and Technology Organisation, Lucas Heights, Sydney, NSW 2234, Australia

4 School of Civil Engineering, The University of Sydney, Sydney, NSW 2006, Australia

5 ISIS Neutron Source, Science and Technology Facilities Council (STFC), Rutherford Appleton Laboratory, Didcot OX11 0QX, UK on ENGIN-X diffractometer at the ISIS neutron source in the UK [1]. The techniques presented here can potentially be applied to other neutron diffractometers, available globally, for the measurement of residual strain in thermal spray coatings, as well as cold spray coatings. Although the physics of neutron diffraction remains the same, the availability of strain measurement instrumentation at different neutron diffractometers varies globally and requires adjustments for a comprehensive strain analysis. Nevertheless, the high resolution of through-thickness non-destructive residual strain analysis, if planned and performed carefully using neutron diffraction, is not normally achievable by other experimental techniques. Except the amorphous phases of materials, this guide can apply to all thermal spray coating and substrate systems to measure through-thickness microscopic residual strain that uses the measurement of the positions of the diffracted neutron spectra peaks to determine changes in the lattice spacing. 
Thermal spray coating processes involve the build-up of a layer of partially molten splats which are heated and propelled by a spray towards a substrate. Difference between coatings obtained through a single pass and multiple passes is shown in Fig. 1(a). The relevant differences are: (a) thicker substrate, (b) higher temperature seen on the substrate, and (c) the substrate is of a different material. Thermal spray processes are characterized by residual strain (or stress) within the coating and substrates due to different physical phenomena (quenching stress, peening effect, deposition temperature, lamella structure) and phase transformations [2-6], (see Fig. 1(b)). That residual stresses dictate the quality and performance (e.g., adhesion, fatigue, tribological behaviour [3]) of the coating-substrate system is a well-established fact. Optimisation of the residual strain field is therefore critical to the life and performance of components, and therefore, measurement of residual stress is crucial to evaluate and quantify coatings quality.

The measurement method dictates the residual stress measured within a thermally sprayed coating (or any other material). Non-destructive (laboratory X-ray, synchrotron $\mathrm{X}$-ray, neutron, Raman spectroscopy, digital image correlation, photoluminescence piezo spectroscopy), semidestructive (hole-drilling \& ring-coring, layer removal, focused ion beam milling, indentation), and miscellaneous other (curvature, modified layer removal, material removal,

(a)

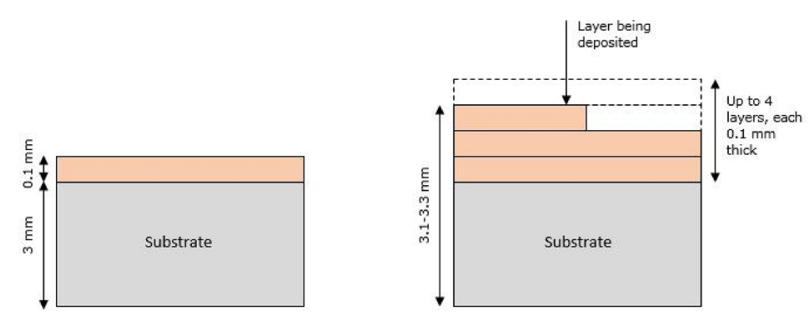

(b)

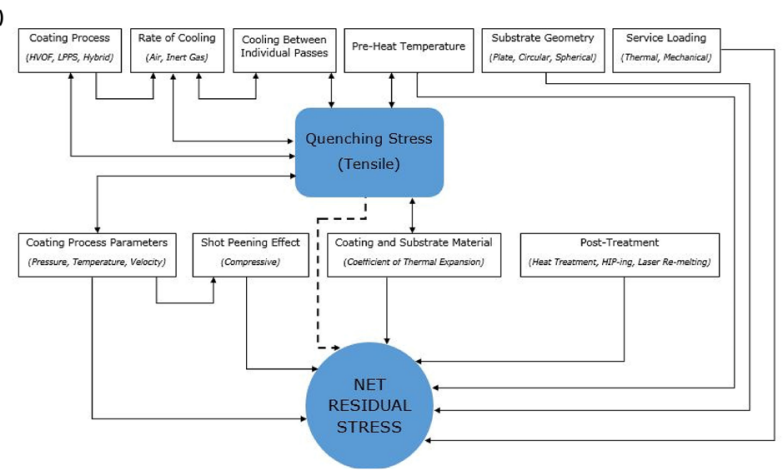

Fig. 1 Thermal spray coating layer build-up and net residual stress: (a) scheme of layer deposition during single and multi-pass thermal spraying, and (c) factor of net residual stress formation in thermal spray coating (adapted from [6]) diametral compression) approaches have been adapted to experimentally evaluate the residual stress fields in thermal spray coatings. The lifetime of the coating system is very much dependent on the residual stress and residual stress values are dependent on the measurement method. This dependency on the measurement method directly influences the verification of numerical and experimental models used to predict the residual stress evolution within the coating systems. Hence what is important and is lacking within the existing literature is the comparative study of the various destructive and non-destructive methods of through thickness residual stress measurement, though studies exist on the use of a single method [4].

Non-destructive measurement of these strain (or stress) fields in relatively thin thermal spray coatings is also technologically challenging, because techniques such as deep hole drilling, laboratory X-ray, and synchrotron X-ray methods are either destructive and/or provide only a subsurface strain measurement. Where the very-near surface residual stresses play a critical role, e.g., surface crack initiation due to wear, surface roughness effects complicate the surface residual stress measurements. Particularly in the case of thermal spray coatings, techniques such as X-ray and synchrotron X-ray can only provide a through thickness residual stress measurement through a destructive process (layer-removal process). Accordingly, recent investigations have concentrated on the use of various non-destructive techniques (e.g., diffraction, optical spectroscopy) for such strain analysis. Comparing the experimental capabilities, based on depth of penetration and depth resolution, Wenzelburger et al. [7] presented an overview about residual stress measurement techniques, measurement principles and characteristic measurement ranges on thermally sprayed coatings and layer composites.

Overall, there are two types of strain diffractometers ((a) monochromatic, where diffraction pattern is a function of the scattering angle, and (b) time-of-flight (TOF) or continuous incident spectrum, where the diffraction pattern is a function of time-of-flight [8]). Neutron sources worldwide (e.g., Africa, Asia, Australia, Europe, and North America) have been used to test strains (and stress) in various materials. In this work, a practical example of the neutron diffraction test method at room temperature and unloaded condition was presented with some of the recent findings of through-thickness residual strain measurements in assprayed coatings via the ENGIN-X neutron diffraction instrument. We also present few examples where thermal spray and cold spray coatings have been characterised at various neutron sources worldwide to study the residual strains and microstructures. 


\section{Origin of Residual Stresses in Thermal Spray Coating}

Residual stresses occur in the material or components at the removal of any external loads (e.g., applied force, displacement, thermal) during manufacturing or service loading [9]. Residual stresses in thermal spray coatings can have different magnitude and distribution, depending on the combination of coating and substrate materials and processing conditions. These stresses can also be altered due to service loading and/or thermal heat-treatment of the coating substrate system. A certain level of compressive residual stress in the coating material is always desirable to resist crack initiation/propagation and coating degradation during service loading. Very high compressive residual stress in coating is however not desired [10-16].

As the coating performance during service loading depends on the structure-property relationships, such as microstructural phases, hardness, fracture toughness, anisotropy, and other microstructural features, it is not always possible to design the optimum residual stress profile in the given coating substrate system. Functional grading of the coating, where the composition of coating microstructure is varied through thickness, either uniformly, or by depositing layers of different materials can sometimes be used to improve the residual stress profile to combat coating delamination. Formation of residual stress is inherent to the nature of thermal spray coating process. These stresses can be caused by quenching stress, peening effect, deposition temperature and phase transformations. Based on physical and metallurgical phenomena dictating the residual strain behaviour, through thickness residual strain profile of thermal spray coating is influenced by the following four major factors [6]:

(a) Role of quenching stress: The quenching stresses, which appear within individual splats, are caused due to the constriction of the individual splat by the lamellae lying underneath that are created due to the preceding spray pass. The degree of constrained contraction is a function of the individual splat temperature and the size of powder particles. Quenching stresses are always tensile as the contraction of splat or lamella during its rapid cooling is constrained by the underlying deposit at relatively lower temperature, means deposited splats thermal contraction after solidification is constrained by the underlying solid splats. Hence, among several factors, the magnitude of quenching stress is function of ratio of the deposition temperature of the powder particle and its melting point.

(b) Peening effect: The high particle velocity seen in certain thermal spray processes is known to have a peening effect which results in compressive residual stresses in the coating. Hence the peening stress is a function of the momentum of the powder particle during thermal spraying, i.e., the deposition velocity and the mass of the powder particle and the temperature-dependent plasticity of the underlying deposit. The mechanism is like the shot-peening of gears and shafts as an engineering practice to induce compressive residual stress to combat fatigue and fracture. Hence, thermal spray coating processes such as the high velocity oxy-fuel (HVOF) process, with relatively high particle velocities are understood to cause relatively high compressive residual stresses in the coatings.

(c) Role of deposition temperature: Macro residual stress occurs due to the differences in the coefficient of thermal expansion (CTE) of coating and substrate materials and the elevated temperature experienced during deposition. These stresses can be compressive or tensile depending upon the mismatch of the CTE of the coating and substrate materials, e.g., if $C T E_{\text {coating }}>C T E_{\text {substrate, }}$, the stress in the coating will be tensile as its contraction is constrained.

(d) Phase transformation: The cooling of impacting powder particle or lamella occurs in a matter of few microseconds from a near-molten temperature to form a solid deposit. This can lead to amorphous phases in the coating material depending upon the temperature of the impacting lamella. There is not enough time for crystal structures to form in a fully molten powder particle, so it is desirable to control the heating of powder particle to near-melting point but not above it. There is also a possibility of oxidation of powder materials in some cases leading to further phase transformations. Some of these phase transformations can lead to a small volume change in the crystal structure. This change in volume can provide an accommodation mechanism at inter-splat boundaries for the coatings, leading to residual strain.

Overall, the residual stress in thermal spray coatings is a function firstly of the distribution of temperature and velocity of individual powder particles in the spray stream. Not all sprayed powder particles have the same temperature or velocity, which is a function of the location of the powder particle within the spray-stream during thermal spraying. Secondly, the residual stress formation is a function of the thermomechanical properties of the coating-substrate system, the most important being the temperature-dependent $C T E$ characteristics and temperature-dependent elastic-plastic behaviour. Additionally, post-spraying residual stress levels are affected by various stress relaxation processes in coating-substrate system, such as plastic deformation, cracking, inter-splat sliding, 
self-annealing, as well as overall compliance of the substrate and coating [17]. Although it is possible to model crudely the evolution of residual stress generation during thermal spraying using numerical modelling, these numerical techniques are able to capture some variation (e.g., the macroscopic gradient from interface to surface due to gradual deposition) of stress or account for phase transformations [15]. Multiscale modelling to combat some of these limitations makes the modelling approach computationally expensive [16]. Hence, non-destructive experimental residual stress measurement remains critical for the evaluation of coating quality and predicting the service life of components.

\section{Principle of Neutron Diffraction}

Introduction to the theory of reflection of $\mathrm{x}$-rays by crystals [18], as well as neutron diffraction and scattering, and how the residual stress can be determined by measuring the spatial variation of the crystal lattice spacing can be explored elsewhere (e.g., Allen et al. [19], Price and Skold [20]). There are number of textbooks available on residual stress analysis by neutron diffraction and residual stress analysis (e.g., Fitzpatrick and Lodini [21], Schajer [22], and Hutchings et al. [23]).

Neutrons interact directly with the nucleus of the atom in a given material instead of the electron cloud which significantly increases the penetration depth of neutrons for strain measurements. In this paper, the emphasis will be on the time-of-flight neutron diffraction technique from pulsed spallation neutron sources. At these facilities which are currently in operation, neutrons of a range of energies are produced at $10-60 \mathrm{~Hz}$ with a short neutron pulse time width typically in the order of 10-100 microseconds depending on the neutron energy and moderator design [24]. The de Broglie's relation given momentum $(p)$ and hence neutron speed $(v)$ is:

$p=m_{n} v=\frac{h}{\lambda}$

where, $m_{n}=1.67 \times 10^{-27} \mathrm{~kg}$ is neutron mass, $h=6.63 \times$ $10^{-34} \mathrm{~J} s$ is Planck's constant. Since neutron travels down the flight path $(L)$ (e.g., $10 \mathrm{~m}$ to $100 \mathrm{~m}$ ), to separate neutrons in time, therefore, time $(T)$ of arrival at the detector is given by:

$T=\frac{L}{v}=\lambda\left(\frac{m_{n} L}{h}\right)$

Neutron diffraction method, as with X-ray diffraction methods, measures the Bragg angle of scattered radiation using the equation [18]: $n \lambda=2 d_{h k l} \sin \theta_{h k l}$

where $n$ is a positive integer, $\lambda$ is the wavelength of the incident wave, $d_{h k l}$ is the inter-planar spacing, $(h k l)$ are the Miller indices, $\theta_{h k l}$ is one half of the angle through which the incident beam is diffracted by planes, which is related to the spacing between crystallographic planes. Hence, peaks are measured at times $(T)$ given after the initial pulse given by:

$T=d_{h k l}\left(\frac{2 m_{n} L \sin \theta_{h k l}}{h}\right)$

Each peak corresponds to an $(h k l)$ family of lattice planes as given by Bragg's law. Like the X-ray diffraction method, the accuracy of the neutron diffraction method is dependent on the knowledge of spacing of the unstressed lattice of the crystallographic planes. This is because the stress is tri-axial at depth [22]. Like the synchrotron X-ray method, spacing at the measurement point in an unstressed lattice should be known but is not easy to measure. The neutron diffraction method shows a smaller range of scattering than X-ray diffraction which is another contributing factor to why it can penetrate deeper. A typical wavelength for neutron diffraction will range between $0.7 \AA$ and $3 \AA$, and can measure the elastic strains in component thickness varying between $0.1 \mathrm{~m}$ and $1.5 \mathrm{~m}$ to less than $1 \mathrm{~mm}$. This means the bulk residual stress within the component can be examined in a non-destructive manner.

Neutron diffraction strain measurements at the UK ISIS Facility utilises ENGIN-X strain measurement diffractometer, which is a pulsed neutron diffractometer equipped with slits and collimators to achieve small gauge volumes [25]. At ENGIN-X, the residual strain in the coating and substrate materials can be obtained from the shift in individual peaks (e.g., shown in Fig. 2) using a single or multiple peak fitting routine. Peaks with little to no overlap with others can be chosen for the strain analysis. There are many different methods for strain-free $\left(d_{h k l}^{0}\right)$ sample preparation and each of them have their own applications depending on the sample microstructure, manufacturing technique and stress field [26]. For thermal spray coatings, the feedstock powder material or the powder obtained by carefully removing the coating from the substrate and crushing it can be used to measure the strain-free lattice parameter $\left(d_{h k l}^{0}\right)$ for the coating material, though these can cause measurement errors. Selection of feedstock powder material can be used only if no compositional or phase change has happened during thermal spraying, and similarly, crushing of coating can be used only if crushing do not induce plastic deformation. In any case, such strain-free powder can then be put in a vanadium tube (which is transparent to neutrons), and its lattice parameter measured. The substrate strain free lattice 


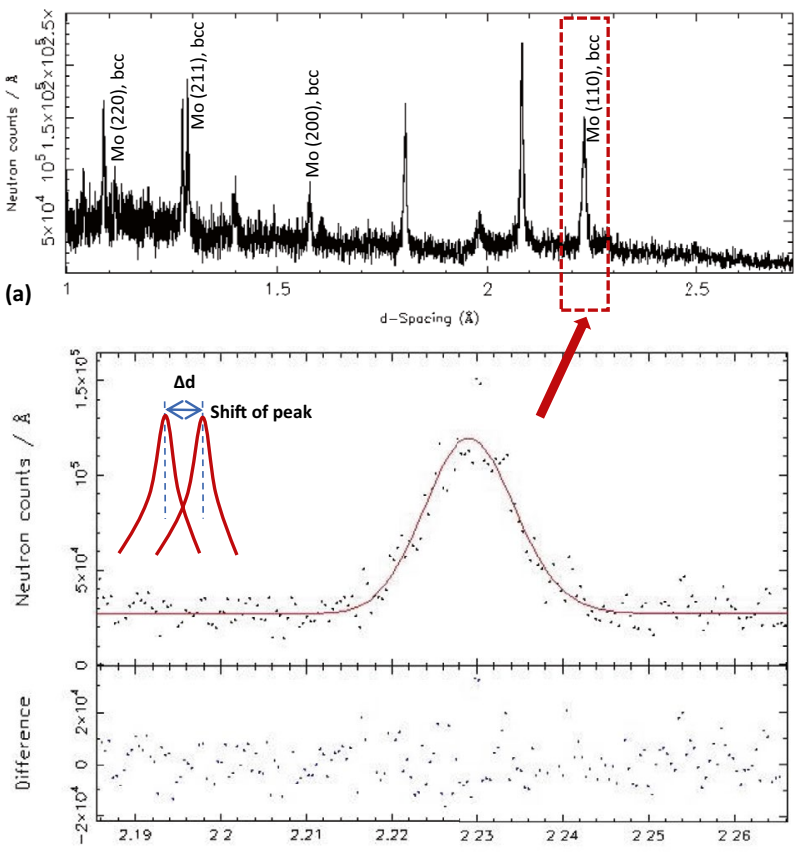

(b)

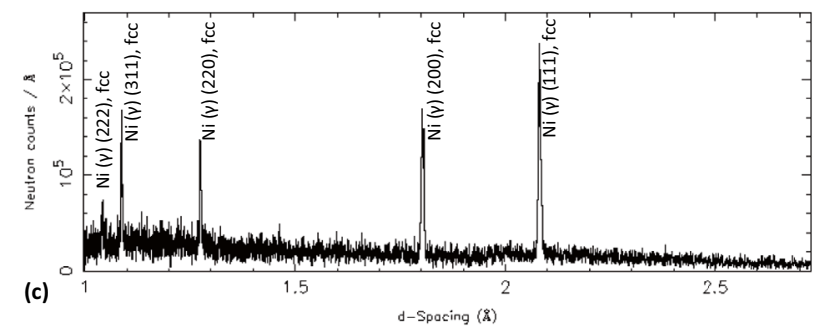

Fig. 2 Typical TOF neutron diffraction pattern near the coatingsubstrate interface in the air plasma spray coating part (including $h \mathrm{kl}$ peaks): (a) $\mathrm{Mo}-\mathrm{Mo}_{2} \mathrm{C} / \mathrm{Al}_{2} \mathrm{O}_{3}$ coating (b) individual peak least-squares refinement of a Mo peak \& showing d-spacing shift scheme, and (c) Hastelloy ${ }^{\circledR X}$ substrate of corresponding $\mathrm{Mo}-\mathrm{Mo}_{2} \mathrm{C} / \mathrm{Al}_{2} \mathrm{O}_{3}$ coating (used as $d_{h k l}^{0}$ for Hastelloy®X substrate). The elastic strain is calculated from the shift in the peak positions defined through a leastsquares refinement (in (b)) [5]

parameter can then be measured at the free surface, only if the substrate has not undergone any plastic deformation. The direct elastic strain (average) in the material at the measured direction can be calculated from the following:

$\varepsilon_{h k l}=\frac{d_{h k l}-d_{h k l}^{0}}{d_{h k l}^{0}}=\frac{\Delta d}{d_{h k l}^{0}}$

where $d_{h k l}$ is the measured interplanar lattice spacing and $d_{h k l}^{0}$ is the stress-free interplanar spacing for the material. The strain is measured in the direction of the scattering vector. The elastic stress can be calculated from these strain values using the through-thickness (coating and substrate) elastic modulus values or Hooke's law:

$\sigma_{i j}=\frac{E_{h k l}}{1+v_{h k l}}\left(\varepsilon_{i j}+\frac{v_{h k l}}{1-2 v_{h k l}} \varepsilon_{q q} \delta_{i j}\right)$

where $\sigma_{i j}$ is stress calculated for a particular direction, $E_{h k l}$ is the elastic coefficient for $h k l$ plane, and $v_{h k l}$ is Poisson's ratio for $h k l$.

\section{ENGIN-X Neutron Diffractometer}

The ISIS spallation neutron sources (monochromatic incident beam), producing approximately $2 \times 10^{18}$ fast neutrons per second as a frequency of $50 \mathrm{~Hz}$ with pulses of $<1 \mu \mathrm{s}$ in width with a $200 \mu \mathrm{A}, 800 \mathrm{MeV}$ proton current impinges on a tantalum (Ta) target [8]. As summarised by Zhang et al. [1], ENGIN-X has a variety of sample equipment to alter the experimental conditions. A specimen mounting stage positions the specimen within the neutron beam path to allow strain measurements. The sample is aligned with the neutron beam with the help of a robotic arm. An in-situ mountable servo-hydraulic stress rig can apply up to \pm 100 $\mathrm{kN}$ cyclic loads to test the samples under various mechanical loading conditions. The rig can be maintained at a low or elevated temperature within the normal atmosphere or under inert gas using a furnace or a cryogenic rig (also called environmental test chamber). The sample mounting stage allows samples weighting up to 1 tonne to be accurately positioned within the measurement point with the accuracy better than $10 \mu \mathrm{m}$. As an example, such mounting stages could be useful to position thermally sprayed large ball valves. The automated experimental setups for complex-shaped samples of various types can be addressed using a laser scanner and robotic arm metrology equipment in combination with the virtual measurement simulation software, SScanSS [27], creating digital geometry.

The practical example of the residual strain test at ENGIN-X neutron diffraction instrument at room temperature and unloaded condition presented here is for air plasma sprayed (APS) Mo- $\mathrm{Mo}_{2} \mathrm{C} / \mathrm{Al}_{2} \mathrm{O}_{3}$ coatings on to Hastelloy$\mathrm{X}{ }^{\circledR}$ substrate [5]. An example of a diffraction peak (scattered by polycrystalline materials [5]) from the instrument is shown in Fig. 2. Time-of-flight (TOF) diffractometers are typically used at pulsed sources (e.g., at ENGIN-X), where each pulse provides a diffraction profile across a large range of lattice spacings. The ENGIN-X instrument has a large flight path from the source to the sample $(50 \mathrm{~m})$ providing $\operatorname{good} \frac{\Delta d}{d_{h k l}^{0}}=50 \mu \varepsilon$ resolution $[8,25]$. 


\section{Test Preparation}

Before carrying neutron strain measurements at ENGIN-X, the preparation involves several steps, as careful planning as possible can help to minimize the losses of beamtime hours or days involved. These include preparation in view of understanding the coating and substrate crystal structure, planning neutron scanning, gauge volume and calibration of the instrument.

\section{Microstructure Assessment and Plan for Neutron Scanning}

Neutron diffraction technique is like X-ray diffraction (XRD), except that the interaction of beam with the matter is different (i.e., X-ray beam interact primarily with the electron cloud surrounding each atom, whereas neutrons beam interacts directly with the nucleus of the atom). This means, neutron beam can penetrate larger depths compared to X-ray beam [28]. Therefore, X-ray diffraction technique has limits in measuring residual strains deep inside the sample (mainly measuring surface and near surface features), while the neutron diffraction can measure residual strains a few centimetres deep inside the sample. It is important to note that neutrons are not a surface or near surface probes [28], but laboratory generated X-ray diffraction technique can be very useful if investigating surface and near-surface residual strain after the coating surface has undergone surface tribological testing (e.g., wear, fatigue).

After assessing the need (near-surface or through thickness residual strain measurement), apart from size and shape of the specimen, it is important to know the material properties and microstructure before neutron scanning using some analytical instruments, such as X-ray diffraction (XRD) for phase analysis, energy dispersive spectroscopy (EDS) for elemental analysis and scanning electron microscopy (SEM) for microstructure analysis. These can be necessary as the thermally sprayed coating may be composed of many crystalline and amorphous structure, and crystalline structures (to be analysed using neutron diffraction) may not be aligned with each other. The XRD analysis for material phases and for crystallographic planes of samples (coating and substrate part) can be very useful in identifying neutron spectra peaks at the ENGIN-X during experimentation. It is important to note that Miller indices for crystallographic planes have notations $(h k l)$, and Miller index is defined as reciprocals of the fractional intercepts that the plane makes with the $\mathrm{x}$-, $y$ - and $z$-axes of the three non-parallel edges of the cubic unit cell. Interplanar spacing between two closest parallel planes with the same Miller indices is designated $d_{h k l}$ [8]. The XRD of the coated surface can show the presence of the dominant phase. If there are distinct textural effects within the microstructure, the infinite orientations criterion of powder diffraction may not be met; in such cases, some of the diffraction peaks might be missing completely and so may not match the reference peaks [5]. However, all major peaks can be identified using the International Centre for Diffraction Data (ICDD) software Powder Diffraction File ${ }^{\mathrm{TM}}$ (PDF) [29] numbers in the analysis.

Microstructure analysis using SEM for both coating surface and cross-section helps to identify the splat orientation or crack and residual defect within the coating layer, including any prior delamination of the coating at the interface. Some understanding of these features (coating layer) and comparison of associated residual strain measurement at ENGIN-X facility may help plan the full experiment with confidence [2,3]. The cross-sectional analysis may also provide exact coating thickness and information about bonding quality between coating and substrate at the interface. If the coating layer has a significantly high number of vertical cracks with layered pores (e.g., normally observed in some YSZ thermal barrier coatings [30]) or the bonding quality between coating and substrate at the interface has already been compromised (i.e., delamination leading to stress relaxation), the residual strain measurement values may not be a true representation of the as-coated sample.

\section{Sample Gauge Volume, Counting Times and Pseudo-strains}

Two important practical considerations are the gauge volume and the counting time for a given sample. How small should be the sample gauge volume for coating part, especially when the coating thickness is typically about $50 \mu \mathrm{m}$ to $500 \mu \mathrm{m}$ ? This is the volume defined as a cuboid of sides $(\Delta V=\Delta x . \Delta y . \Delta z)$. In neutron strain experiments, the diffracted signal is obtained from a defined volume [31] in space that contains part of the specimen material. Typically, some understanding of the structure and size of original sprayed powder (e.g., conventional, nanostructured) and through-thickness splat size can help define the gauge volume. The sketch in Fig. 3 shows scheme of through thickness strain measurement on disc shaped coated sample, and sample location between detectors presents how a sampling volume is defined through apertures (e.g., slits) placed in the incident and diffracted beams. The intersection of the incident and diffracted beams defined by slits and collimators defines the gauge volume [25]. Usually, the largest gauge volume can minimise the counting times (useful for bulk or large volume of material testing). However, different coating and substrate materials will require different counting times depending on the sample geometry, dimensions, and measurement point positions due to the neutron path 
(a)

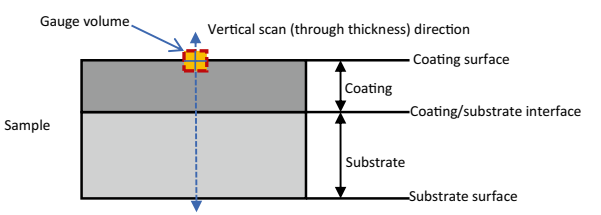

FRONT VIEW

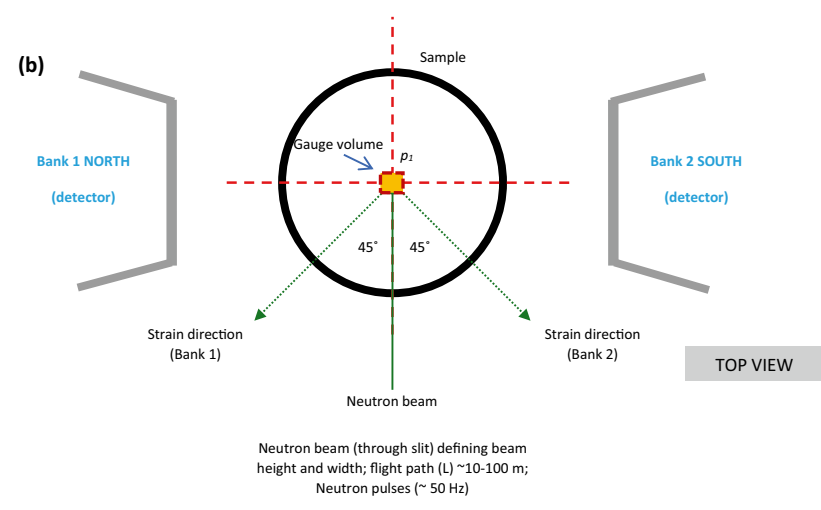

Fig. 3 Scheme of through thickness strain measurement on disc shaped coated sample and sample location at ENGIN-X: (a) front view of sample cross-section, and (b) top view of sample location between detectors

lengths. Larger gauge volumes can be used to reduce counting times, but it needs to be optimised between counting time and measurement spatial resolution. The diffracted signal recorded on the detector (e.g., at Bank 1, Bank 2; centred at $\pm 90^{\circ}$ to the incident beam) in neutron strain and stress measurements originates from this volume [3]. In thermally sprayed coating sample testing, the gauge volume can be rastered across the sample through thickness (vertical scan mode [2-5]), e.g. Figure 4(a,b), as the divergence perpendicular to the diffraction plane, does not affect the resolution. ENGIN-X uses a few sets of removable radial collimators, such as $0.5 \mathrm{~mm}, 1 \mathrm{~mm}, 2 \mathrm{~mm}, 3 \mathrm{~mm}$, and $4 \mathrm{~mm}$ gauge width.

As demonstrated in previous work [5], the scattering trials can be conducted in a vertical scan mode with a slit gap of $200 \mu \mathrm{m}$ (or $0.2 \mathrm{~mm}$ ) to determine the through-thickness residual strain profile of the coating-substrate system. To achieve a high-resolution profile of the through thickness strain, a submerged beam near the coating surface can be used. The same can be done with the coating and substrate near the interface. As an example, a gauge volume of $0.2 \mathrm{~mm} \times 8 \mathrm{~mm} \times 4 \mathrm{~mm}$ can be employed. The $0.2 \mathrm{~mm}$ gauge size can be defined along with the coating build-up direction necessary to maintain sufficient spatial resolution. The other dimensions can be increased up to $8 \mathrm{~mm} \times 4 \mathrm{~mm}$ in the regions or directions where there are small strain gradients and minimal microstructure variations to minimise counting times. This is necessary to ensure that the data point collection strategy is optimised for the beam time available.

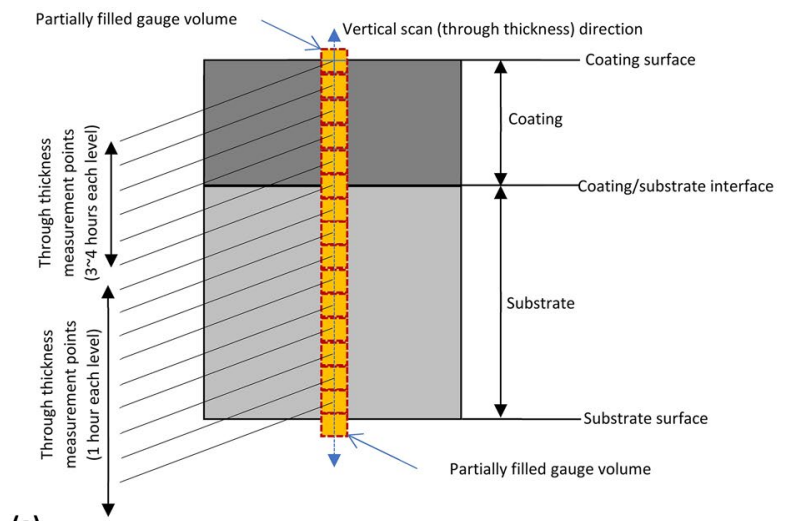

(a)

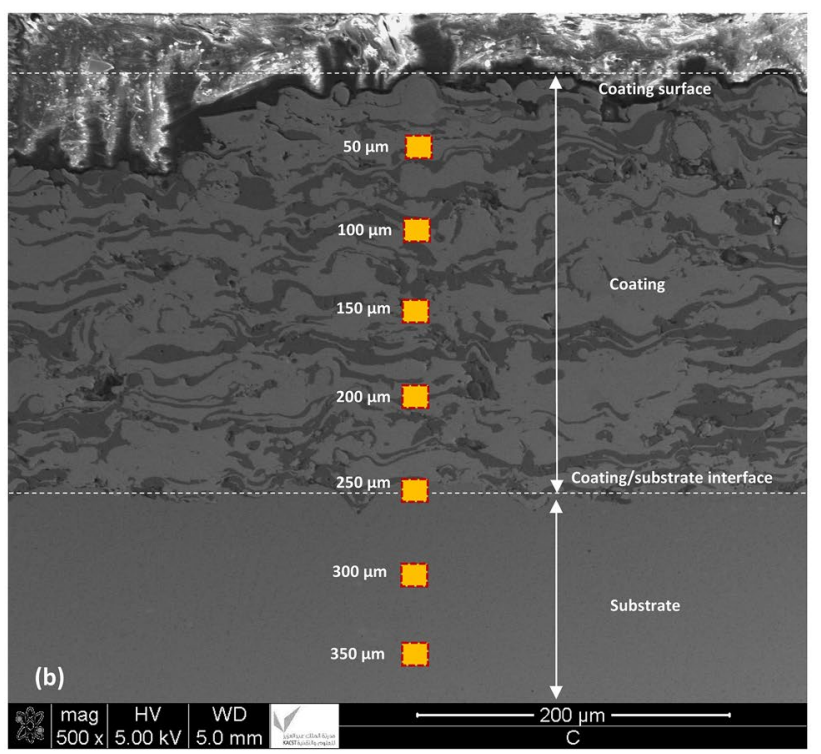

Fig. 4 Gauge volume as seen vertically (for d-spacing measurement): (a) recommended scheme, and (b) cross-section image of $250 \mu \mathrm{m}$ thick $\mathrm{Mo}-\mathrm{Mo}_{2} \mathrm{C} / \mathrm{Al}_{2} \mathrm{O}_{3}$ coating on $4.7 \mathrm{~mm}$ thick Hastelloy ${ }^{\circledR X}$ substrate (showing features near the surfaces and interface and practical measurement positions from top of the coating surface)

There are number of studies on pseudo-strains induced by surface-effects, e.g., by Suzuki et al. [32], where it has been demonstrated that pseudo-strains appear in throughsurface strain measurements when the gauge volume is incompletely filled by the sample. It has been indicated that tensile pseudo-strains due to the surface-effect increases near the sample surface and exhibit a similar trend regardless of the size of the gauge volume, while the pseudo-strains are larger for smaller gauge volumes [32]. However, there are no pseudo-strains when scanning vertically out of or away from a horizontal surface because there is no change in the diffraction angle [6]. Therefore, through thickness vertical scan has been implemented in the current work on thermal spray coatings to avoid pseudo-strains when scanning at or near to the sample surface with a partially filled gauge 
volume (Fig. 4(a)). The specimen position can be adjusted using three orthogonal motors along the three-co-ordinate axis (X, Y - Horizontal, Z - Vertical). The specimens can be moved along the $\mathrm{Z}$-axis along steps of $50 \mu \mathrm{m}$ within the coating and in steps of $25 \mu \mathrm{m}$ within the substrate material (i.e., closer to the interface). A beam height of $0.2 \mathrm{~mm}$ can be achieved by passing the beam through an assembly of horizontal and vertical slits. Partially submerging the gauge volume allowed the strain in the coated material with coating thickness $250 \mu \mathrm{m}$ (Fig. 4(b)) to be measured, whereas a fully submerged beam can be used to measure the substrate strain. To measure the strain within the coating and substrate, different measurement times will be necessary (e.g., 3 to $4 \mathrm{~h}$ per vertical step along coating depth and coating-substrate interface), and fully in substrate (e.g., 30-45 min per vertical step along substrate depth) [2-6]. However, it is important to note that these measurement times are not universal and could vary from sample to sample (i.e., coating-substrate system).

\section{Calibration of a Neutron Diffractometer}

The calibration of the neutron diffractometer and associated instruments must be tested prior to scanning (normally carried by instrument scientist) using the procedure that has been verified according to practices at ENGIN-X. A sample configuration that produces statistically determinate strain in the sample must be used. The procedure must be repeated to ensure checking of the measurement data. Calibration of the ENGIN-X neutron diffraction instrument is done through the determination of the wavelength of the incident neutron beam and the angular response of the neutron detector accurately [8]. At a TOF (e.g., ENGIN-X) source, isotropic neutron scatterers (e.g., vanadium) with very weak or no diffraction peaks of their own are used to perform the necessary calibration of the detector efficiency as a function of wavelength [33].

\section{Strain Testing}

Strain testing includes experiment preparation in view of sample mounting, reference point, fast scanning, full scanning of sample, and scanning for $d_{0}$-spacing $\left(d_{h k l}^{0}\right)$.

\section{Sample Mounting}

Sample positioning on stage is an important element of neutron diffraction strain measurement. In thermally sprayed coating sample investigations, the objective could be to measure the distribution of strains through-thickness within the specimen (Fig. 4(b)), however, there could be other analysis as well. Hence, a sample positioning on stage should enable the change of location of the gauge volume within the sample. In some cases, a dedicated sample holder for mounting on the table may be needed, and this should be designed and built-in advance in discussion with instrument scientist before visiting the ENGIN-X lab for experiments.

The Fig. 5 shows the test set-up (coated sample location between detectors, vanadium tubes filled with coating powder form, removable horizontal slit, and fixed vertical collimator (slit) with options to vary gauge width). As shown in Fig. 5(a), the ENGIN-X positioner where samples are mounted is a high-capacity translation and rotation stage for 1-tonne maximum specimen weight (horizontal motion: $\pm 250 \mathrm{~mm}$, vertical motion: $600 \mathrm{~mm}$, rotational about a vertical axis: $370^{\circ}$ ). Samples can be mounted with reproducible positioning on these breadboards using a LOCOMETRIC Precision Sample Mounting System [34]. If the experiment requires testing at a given temperature and external mechanical loading conditions, the servo-hydraulic stress rig and environmental test chamber (e.g., furnace or cryogenic, which can be varied at different rates) within the normal atmosphere or under inert gas, can be useful. The test samples could be mounted in loading fixtures (with a pre-stress) for sequential loading through a range of force and temperature as mentioned above. The change in interplanar d-spacing can then be measured for each force and temperature conditions $[1,33]$.
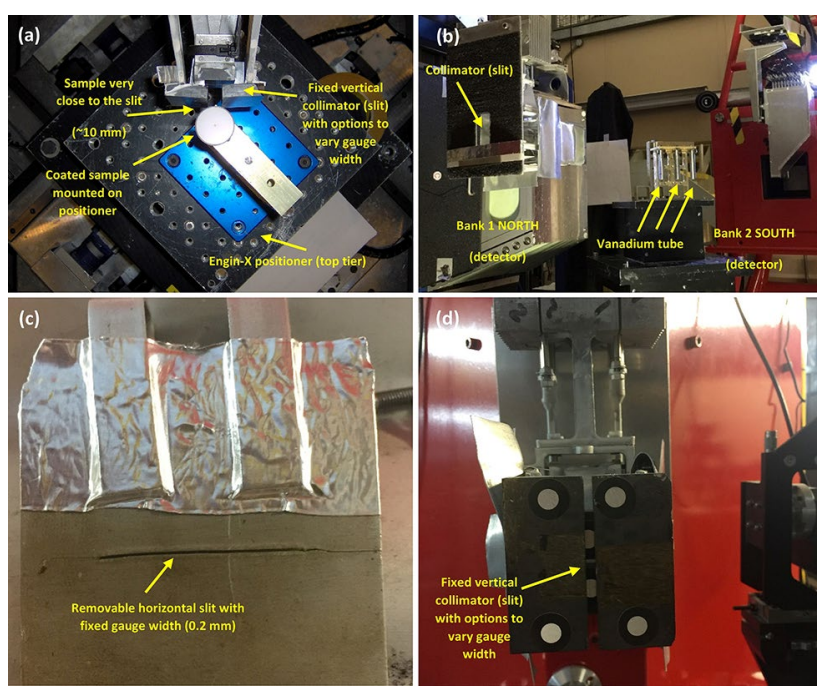

Fig. 5 Test set-up and measuring residual strain using neutron diffraction at ENGIN-X: (a) top view of coated sample location between detectors, (b) showing vanadium tubes filled with coating powder form, measuring the strain-free lattice spacing, (c) removable horizontal slit, and (d) fixed vertical collimator (slit) with options to vary gauge width 


\section{Reference Point and Fast Scanning}

These are steps for the strain measurements. It is good practice to position the centroid of the gauge volume at the reference point. The ENGIN-X instrument reference axis and neutron beam reference height must be aligned at the centre of the instrument gauge volume (IGV) [8]. This is to refer to the position of the test sample. One practical method is to first centre a vertical pin on the table (ENGIN-X positioner, top tier) so that pin tip defines the reference point. The centring of the pin tip can be done by viewing the tip through a theodolite, which is an optical apparatus for measuring coordinates of visible points. The centring of the pin tip can also be done by using a micrometre contact gauge and adjusting the position of the translator. Following the centring steps, the gauge volume is arranged to have the reference point as its centre by positioning the apertures in the horizontal and vertical planes [8]. Once the sample has been mounted on the stage or table (ENGIN-X positioner, top tier), the necessary step in performing neutron diffraction of the effective position in the sample at which the strain is measured is important. This is typically done through fast scanning (using vertical scan mode, through-thickness of the coated sample) of thermally sprayed coatings. These steps (reference point, fast scanning) can be adjusted to ensure that the centre of the neutron spectra intensity profile versus the vertical position of the translator is in the encoder values corresponding to the reference axis position [8].

\section{Scanning of Sample for D-spacing}

The experiments are typically conducted in vertical scan mode (Fig. 4) to measure the through-thickness residual strain profile of the coating-substrate system. Several samples can be assembled on a holder. The details of the vertical scan method are described elsewhere for thermal spray coatings [2-6]. Strain measurements are generally performed at the centre of the specimen (at $p_{1}$ ) but can also be performed at other locations (e.g., at a radial distance from the centre [3]), as shown schematically in Fig. 3(b). Although the measurement locations in the specimen can be changed, the distance to the detectors (Bank 1 and Bank 2) is always the same. A partially submerged beam can be used at the coating surface, whereas a full beam submerged partly in the coating and the substrate materials can be used at the coating-substrate interface to obtain a high-resolution profile of the through thickness residual stress distribution. For the case of beam submerged at the coating-substrate interface, simultaneous measurements can be made in the coating and substrate materials. The gauge volume calculations for both materials in this case are based on principles similar to that of partially submerged beam. Measurement of the residual strain at a volume smaller than the gauge volume using a partially submerged beam can be understood from other studies [25]. The coating-surface interface and the coating surface can be located within a resolution of $50 \mu \mathrm{m}$ with the help of fast vertical scans and careful location of the specimen using theodolites.

\section{Scanning of Sample for $d_{0}$-spacing}

Either removing the coating from the substrate and crushing to create the coating powder or direct measurement of the feedstock powder can be carried out to measure the strain-free lattice parameter $\left(d_{h k l}^{0}\right)$ [2-6], though each of such methods would have limitations and challenges. This powder can then be put in a vanadium tube and its lattice parameter measured, as shown in Fig. 5(b). The strain-free lattice parameter for the substrate can be measured at the suitable area of the uncoated substrate sample where $d_{h k l}^{0}$ has been verified to be independent of position and direction at that region in the materials [8] (or unsprayed substrate can also be used). In case dedicated reference samples are needed; these should be prepared in advance before visiting ENGIN-X lab for experiments or while other measurements are ongoing.

\section{Strain and Stress Analysis}

\section{Pre-processing Steps}

Neutron spectrums are characterised by several parameters that can be analysed, e.g., position/shape/amplitude/width of the peak in the spectra, however, the positions and widths of the spectra are important parameters which can be related to the strain field in the sample [8]. Typical steps after neutron diffraction measurement of coated samples include plotting spectra (neutron counts v/s d-spacing; neutron counts $\mathrm{v} / \mathrm{s}$ TOF, e.g., Fig. 2, and as an example, a set of experimental raw data is provided in Appendix A, using ISIS ENGIN-X software Open genie) for both banks (detectors) for each location. It is highly recommended to carry few preliminary analyses as soon as the first few neutron spectra data is available during the experimentation. This can assist in the identification of neutron spectra peaks (corresponding to each $h k l$ planes known or identified through X-ray diffraction carried for test preparation) in both banks, and in locating depth where they had no or minimal overlap with other peaks. If one single or several non-overlapping peaks are measured, their spectra parameters are analysed for each peak separately, however, the analysis of the whole spectrum can be performed using Rietveld methods. 


\section{Post-processing Steps}

The post-processing steps include a listing of $d_{h k l}$ and $d_{h k l}^{0}$ spacing for each run number and bank number (both in coating and substrate part) in a sample. The analysis of $d_{h k l}$ and $d_{h k l}^{0}$ spacing in diffraction peaks for the coating and substrate materials is possible either (or both) using the Rietveld refinement method or individual peak method. Use of the Rietveld refinement method may be easy, however, in the individual peak method, the listing of $d_{h k l}$ and $d_{h k l}^{0}$ spacing needs to be done for each identified peak for peak-by-peak analysis. The peak-by-peak analysis can help in analysing the strain for each $h k l$ planes.

Evaluation of the lattice parameters in the case of simple crystal structures can be carried out through the Rietveld refinement analysis which considers multiple peaks in the spectra. However, where the spectra have overlapping peaks, e.g., coating to substrate and substrate to coating, this method can be challenging to apply. At each measurement location, the neutron spectra recorded by the individual detectors are time focused using the computer code GSAS [35]. Using a library of common engineering materials, this software automatically refines the single-peak and fullspectra within the diffraction spectra [25]. To ensure the convergence of the refinement, single-peak refinement of the most intense peak is performed to estimate the lattice parameters initially.

\section{Strain Calculations}

\section{Rietveld Profile Refinement}

The standard method of analysing the neutron diffraction experimental results is the Rietveld profile refinement method. In this, the intensity measured as a function of the scattering angle is fit with the parameters of a model. The strain can be obtained from the shift in diffraction peaks for the coating and substrate materials using Rietveld refinement. The Rietveld method allows analysis of spectra with strongly overlapping lines and patterns from multiphase materials [36]. A change in d-spacing or $\Delta d=\left(d_{h k l}-d_{h k l}^{0}\right)$ in the lattice spacing due to internal strains will result in a shift of a Bragg peak position $\Delta \theta$ (typically in the range of $0.005^{\circ}$ to $0.2^{\circ}$ ) when a single wavelength is used [8]. The General Structure Analysis System (GSAS) software [35] can be used in the data analysis.

\section{Individual Peak Fitting}

The strain can also be obtained from the shift in 'individual' diffraction peaks for the coating and substrate materials using a single peak fitting routine [23] using GSAS software.
The fitting parameters of the refinement, confirming good fit to the diffracting pattern (shown in Fig. 2(b)). The peaks chosen for the strain analysis should be such that they had no or minimal overlap with other peaks. As an example, a set of experimental raw data is provided in Appendix A (for RB1510238 [5]).

\section{Strain Calculations}

After analysing individual diffraction peaks results, residual strain $\left(\varepsilon_{h k l}=\frac{d_{h k l}-d_{h k l}^{0}}{d_{h k l}^{0}}\right)$ results in coatings and substrate can be analysed using individual peak method or Rietveld (using equation (5)). Figure 6 shows an example of residual strain measurement (plotted using single peak fitting routine analysis). The averaging of strain across the various identified crystallographic planes can also give similar results to the Rietveld refinement.

\section{Stress Calculations}

The average coating and substrate residual strain (and stress) can vary significantly with the coating conditions. From the measured strain, the residual stresses can be determined using the elastic constant (Hooke's law, equation (6)) for
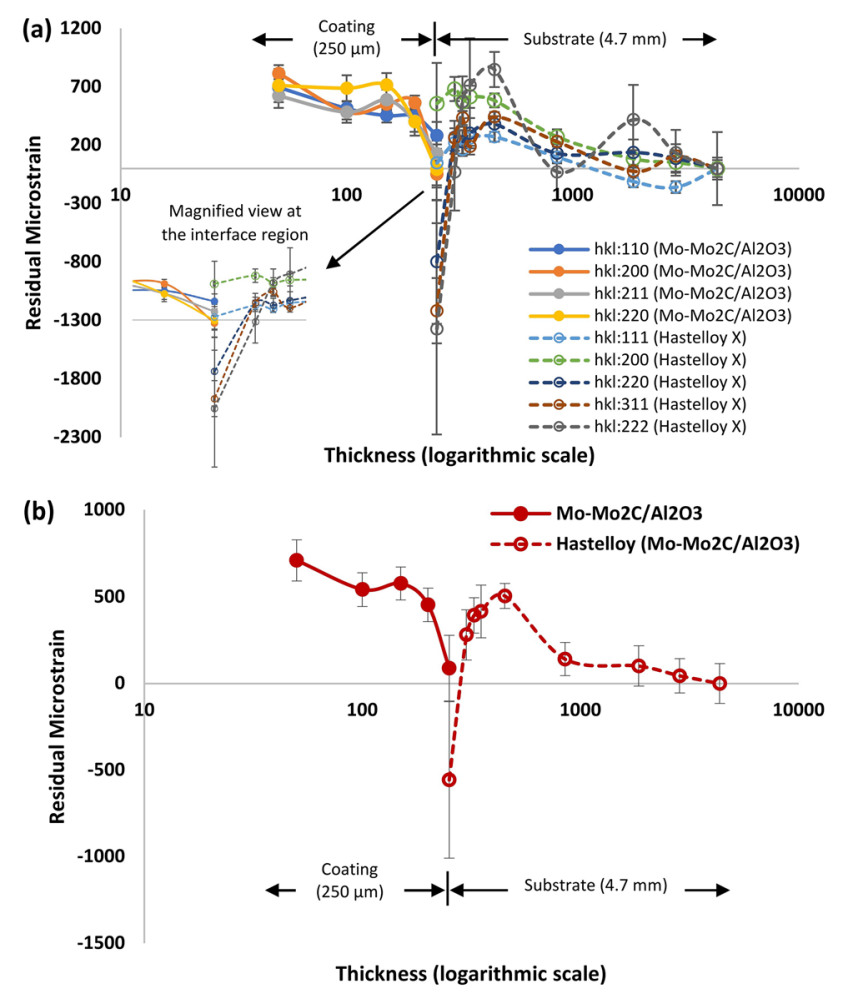

Fig. 6 Residual strain analysis based on single peak fitting routine analysis for neutron diffraction residual strain in $\mathrm{Mo}-\mathrm{Mo}_{2} \mathrm{C} / \mathrm{Al}_{2} \mathrm{O}_{3}$ ( $250 \mu \mathrm{m}$ thick coatings on $4.7 \mathrm{~mm}$ thick Hastelloy®X substrate): (a) for individual peaks, and (b) average of all individual peaks [5] 
material types, where bulk elastic modulus values of materials are typically used from literature, or through standard mechanical testing of samples (e.g., bending, or tensile tests) or can be calculated from single-crystal constants [37] using the Eshelby-Kroener model [38], as demonstrated by Keslar et al. [39], though each of such methods would have limitations and challenges.

Alternatively, as shown in Fig. 7(a), number of nanoindentation based elastic modulus measurement for stress measurement $(\sigma=\varepsilon E)$ can be done on each coatingsubstrate cross-section, which can be dispersed in five lines of 5 measurement points each, at a specific space from the boundary (i.e., coating surface). Indentations can be spaced
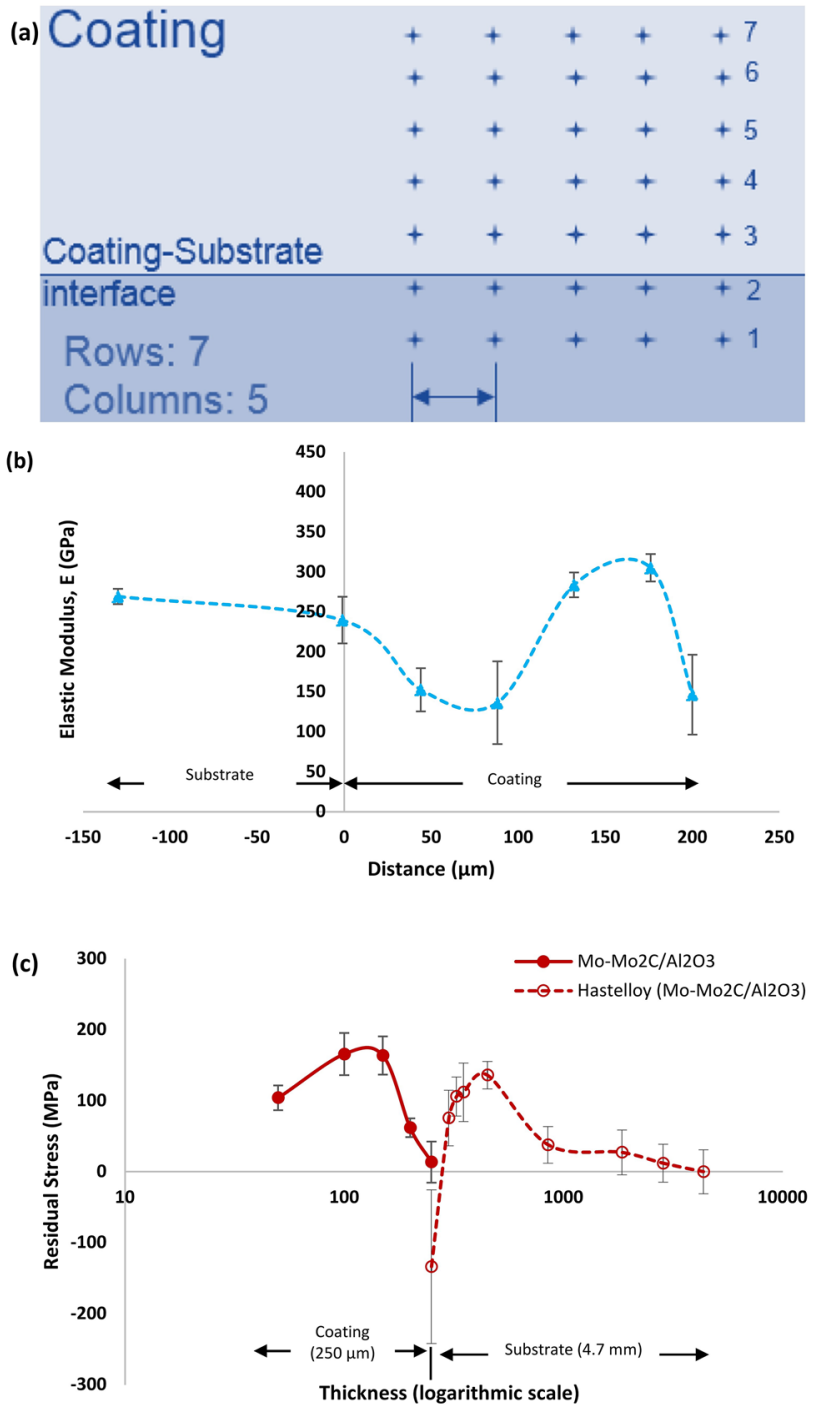

Fig. 7 Residual stress analysis: (a) scheme of nanoindentation array (coating-substrate cross-section surface) to measure elastic modulus (b) for residual stress analysis, and (c) residual stress analysis based on average of all individual peaks in $\mathrm{Mo}-\mathrm{Mo}_{2} \mathrm{C} / \mathrm{Al}_{2} \mathrm{O}_{3}(250 \mu \mathrm{m}$ thick coatings on $4.7 \mathrm{~mm}$ thick Hastelloy ${ }^{\circledR X}$ substrate) [5] by several microns apart, to avoid any interaction of adjacent indentations. By the same method, some 10 measurements can be spread in two lines of 5 measurement points apiece on each substrate cross-section near the interface, at a specific distance from the interface. To convert the through-thickness residual strain data to analyse the corresponding residual stress distribution, an average value of measured elastic modulus can be utilized where the measurement depth location of residual strain and elastic modulus did not match. As an example, a set of experimental data and analysis is provided in Fig. 7(b), and raw data is provided in Appendix A. Considering the applicability of such nanoindentation data to relate neutron diffraction residual strain to stress, it can be stated that the gauge volume for residual stress measurement comprises a polycrystalline material and stress is averaged across crystals in a given direction. The residual stress is also affected by the coating defects such as porosity, cracks, imperfect bonding, etc. Although the volume of material displaced in the elastic-plastic deformation during nanoindentation can be relatively larger when compared to the gauge volume of the beam, especially when smaller gauge volumes are used in thinner coatings, nevertheless, both the stress and nanoindentation measurements average the response of the material along with its defects. Hence, this technique has been used in the published literature to relate strain to stress [e.g., 2-6]. Other techniques of elastic modulus measurements such as tensile testing of coating substrate material or coating removed from the substrate are less accurate as they do not show local variations in material which changes as coating thickness develops, it is difficult to estimate the modulus contribution from the coating and substrate in the specimen, and for the cases of coating removed from the substrate, the residual stress in the coating changes and hence reduces the accuracy of both the modulus and residual stress measurements. It may be possible to get a local through thickness modulus of the coating volume using in-situ diffraction measurements by physically straining the coating material, however it has not yet been attempted in thermal spray coatings. As for the variation in the elastic modulus, the standard deviation of the values shows the local variations in the coating material defects. Also worth noting is that the residual stress variations also influence the modulus values by altering the stress distribution during nanoindentation.

If required, the stresses can then be normalised by dividing by the yield stress data $\left(\frac{\sigma}{\sigma_{y}}\right)$ of the coating and substrate materials, respectively. In the example shown (Fig. 8), the yield stress of the Hastelloy- $\mathrm{X}{ }^{\circledR}$ substrate is taken as $385 \mathrm{MPa}$ and for $\mathrm{Mo}-\mathrm{Mo}_{2} \mathrm{C} / \mathrm{Al}_{2} \mathrm{O}_{3}$ it is taken as $770 \mathrm{MPa}$ (at zero plastic strain) [40]. It is important to note that the yield stress value for coatings considered are for bulk materials with limited relevance to such coatings. However, 


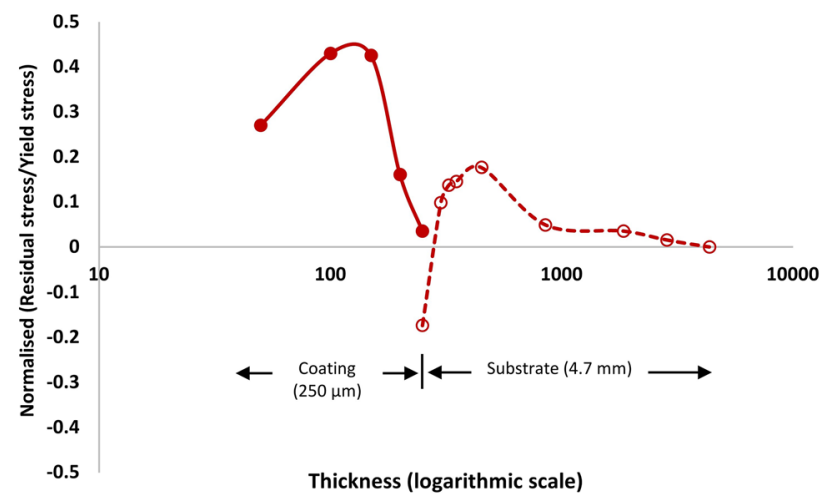

Fig. 8 Normalised stress analysis based on average of all individual peaks in $\mathrm{Mo}-\mathrm{Mo}_{2} \mathrm{C} / \mathrm{Al}_{2} \mathrm{O}_{3}(250 \mu \mathrm{m}$ thick coatings on $4.7 \mathrm{~mm}$ thick Hastelloy ${ }^{\circledR} \mathrm{X}$ substrate) [note: yield stress of the Hastelloy- $\mathrm{X}{ }^{\circledR}$ substrate is taken as $385 \mathrm{MPa}$ and for $\mathrm{Mo}-\mathrm{Mo}_{2} \mathrm{C} / \mathrm{Al}_{2} \mathrm{O}_{3}$ it is taken as $770 \mathrm{MPa}$ (at zero plastic strain)]

considering limited relevance, as shown, both for coating and substrate parts, the normalised stress $\frac{\sigma}{\sigma_{y}}<1$.

\section{Precision and Bias}

Neutron scattering allows the atomic positions in the structure to be determined with high precision. At ENGIN-X, the precision of neutron diffraction instrument alignment within $50 \mu \varepsilon$ can be achieved [25]. The accuracy of this method is considered to be absolute, however, the error or uncertainties can be related to various factors, such as quality of the diffracted neutron peaks (background, noise), calibration, failure to consider appropriate time during the measurement to obtain accurate scattering intensity and the peak positions. Other factors could be variation in temperature or chemical composition of the sample as a function of position and time, including measurements if performed in regions with steep strain gradients (e.g., coating-substrate interface) can also add to error, leading to uncertainty in strain.

Considering issues related to steep residual strain gradient at coating-substrate interface, the authors have looked at numerous coating-substrate materials, i.e., neutron diffraction residual strain analysis on thermally sprayed coated samples (e.g., HA: $\mathrm{Ca}_{10}\left(\mathrm{PO}_{4}\right)_{6}(\mathrm{OH})_{2}[2], \mathrm{Al}_{2} \mathrm{O}_{3}[3$, 41], WC-10\% NiCrBSi [4, 6], $\mathrm{Mo}-\mathrm{Mo}_{2} \mathrm{C} / \mathrm{TiO}_{2}$ [5], Mo$\mathrm{Mo}_{2} \mathrm{C} / \mathrm{ZrO}_{2}$ [5], $\mathrm{Mo}-\mathrm{Mo}_{2} \mathrm{C} / \mathrm{Al}_{2} \mathrm{O}_{3}$ [5], YSZ [41], and WC$12 \% \mathrm{Co}[42])$ and have analysed strain gradients. It can be acknowledged that coating-substrate material and thermal spray processing techniques (with varying degree of temperature and particle speed) influences the stress field and their interpretation. At the coating-substrate interface, the discontinuity in the stress field is typical of overlay coatings, which is either due to the mismatch of thermal and mechanical properties of the coating and substrate materials, or because coating is typically applied in molten state or at elevated temperature. The zone at coating-substrate interface along with the introduction of phase changes within the coating material further complicate the stress field. Within the substrate material, however, the residual stress at the coating substrate interface can be close to zero with a small gradient of tensile stress through its thickness. Though this may be specific to this study, e.g., [6], there is no generic way of determining what this profile will look like without modelling (e.g., analytical, or finite element methods), both of which still require experimental results for validation, for which neutron diffraction is useful method [16]. Meanwhile, these low values of tensile residual stress are indicative of the low substrate heating during thermal spraying. The near zero value of residual stress within the substrate at the coating substrate interface, instead of the expected tensile stress is indicative of the influence of compressive residual stress due to grit-blasting prior to thermal spraying, which was attenuated due to the differences in the CTE during the coating process, or (partially or completely) annealed due to high temperature during spraying [6].

Usually, steep strain gradients occur at surface or at interfaces and the test requires the gauge volume to be partially submerged [43]. It is to be appreciated that by using through thickness vertical scanning technique, potential errors in strain, caused by partial filling of the gauge volume introducing a geometric shift in the diffraction peak position (pseudo-strains) can be avoided [6]. It is important to note that it normally comes down to either carry single peak analysis of neutron spectra in complex material cases where we lose strain in lattice translational vectors and measure strain only in one direction, whereas in simple material cases it is possible to use multiple peaks of neutron spectra to get strain in the entire lattice. Even for the cases where multiple peak analysis is applied, issues could remain at the interface. Experimentally, by using the fast vertical scan and careful levelling of specimen using theodolites (for strain test set-up), the coating surface, and the coating-substrate interface can be located with a resolution of about $50 \mu \mathrm{m}$ [2]. This process indicates some level of positional accuracy with which measurement points within the sample can be located. When the gauge volume is partially submerged, a smaller gauge size is obtained than is defined by the aperture size alone, as detailed elsewhere by Fitzpatrick and Lodini (2003) [21]. 


\section{Examples of Neutron Diffraction of Thermal Spray Coatings at Other Facilities Worldwide}

Considering source types, various monochromatic diffractometers are NECSA/MPISI, HANARO/RSI, BARC/(PD-3, SANS), CAEP/RSND, BATAN/DN1, PINSTECH, ANSTO/ (KOWARI, ECHIDNA, QUOKKA, KOOKABURRA), ILL/ (SALSA, D20), LLB/DIANE, HZB/E3, GEMS/STRESSSPEC, BNC/ATHOS, INR/DIR-1, IAM/(HB4, HB5), PNPI/ ARES, NPI/(TSKN-400, HK4), NIST/(BT8-DARTS, SANS $30 \mathrm{~m}$ NG3), ORNF/HB-2B NRSF, and various time-of-flight diffractometers are JAEA/TAKUMI, ISIS/ENGIN-X, SINQPSI/POLDI, FLNP/( EPSILON-MDS and SKAT, FSD), ORNF/VULCAN, LANSCE/(HIPPO, SMARTS), and there may be other sources. There are not many, but good number of examples where thermal spray coatings have been characterised to study strains as well as microstructures (e.g., porosities, volumetric and size characterisation of void morphologies, texture) using neutron diffraction techniques. As described above, the ISIS/ENGIN-X neutron facility has been used to measure residual strain in thermal spray coatings [e.g., $2-6,11-15,41]$, the methodology of which has been summarised in practical steps above. This section however presents some examples and general assessment, and by no means presents a comprehensive critical review on the application of neutron scattering techniques to study thermal spray coatings.

\section{Overview of Test Protocols At Neutron Diffractometers}

The protocols for neutron diffractometers are dependent on the type of diffractometer. Although all diffractometers use Bragg's law of diffraction $\left(n \lambda=2 d_{h k l} \sin \theta_{h k l}\right)$, the way it is applied varies depending upon the instrument. Neutron diffractometers can therefore be broadly classified as (a) monochromatic, where $\lambda$ value is fixed and angle $\theta_{h k l}$ varies to diffract a given material phase or peak, and (b) time-offlight, where $\theta_{h k l}$ value is fixed at $90^{\circ}$ and $\lambda$ value changes for measurement. As listed in Table 1, majority of the diffractometers operate with monochromatic incident beam, where the diffraction pattern is a function of the scattering angle, however, few diffractometers operate with choppers to create a pulsed regime where the diffraction pattern is a function of time-of-flight (TOF) [8]. While both diffractometer types could be complimentary to each other, there are high level similarities in test protocols at both diffractometer types in strain measurement of thermal spray coatings. As an example, for strain imaging using TOF diffractometer type (e.g., ENGIN-X at ISIS), work by Santisteban et al. [25] can be cited, whereas, for strain imaging using monochromatic diffractometer type (e.g., SALSA at ILL) for near surface or interface stresses, work by Pirling [44] can be cited.
Overall, the underlying common test protocols of strain determination using both diffractometer types include direct measurement of the lattice plane spacings (or $d_{h k l}$ ) using Bragg's law, and then determination of the corresponding strains by referencing against the strain-free $\left(d_{h k l}^{0}\right)$ condition and then converting to stresses by incorporating the elastic properties of the materials. It has been suggested that the total neutron flux at a TOF diffractometer is comparable with the monochromatic flux at a conventional diffractometer, but the intensities of the individual peaks are much lower owing to spectrum distribution over the wide wavelength range [8].

It is important to note that both single and multiphase materials can be successfully investigated using either monochromatic or TOF sources. However, the key difference between both diffractometer types is that for monochromatic types, the strain analyses are done from single Bragg peak assessments (typically selecting dominant phases in coatings with adequate intensity), whereas for TOF types, the strain analyses can be done from multiple Bragg peak assessments (can select all phases in coatings with adequate intensity). Considering estimation of texture and anisotropy of materials, usage of TOF instrument provides information on several peaks, which means analysis of multiphase materials is straightforward and simultaneous measurement is possible [8].

Other protocols or practices to consider while carrying measurement at both diffractometers types are that related to gauge volume (defined by the incident beam collimator and the detector collimator), through thickness (coating and substrate) vertical scan (scanning through flat surface or interface to correct for pseudo-strain), sample orientations (in the plane and perpendicular to the plane of the coating), finding surface through vertical scan, sensitivity to surface roughness for near surface measurements (could be challenging unless using an extremely small gauge volume and the surface roughness is enough to cause pseudo-strains), accuracy when measuring near the surface or edge (depending on the size of the gauge volume), spatial resolution (diamond shaped cross-section of the gauge volume could help scan closer to the surface or edge), types of detectors or detector position sensitivity (typically monochromatic types have angular coverage and can measure one or few peaks at a time), additional crystal reflections to avoid overlapping of peaks from different phases present in the gauge volume, and from multiple wavelengths incident on the specimen (for monochromatic types).

\section{Strain Analysis}

As will be seen through examples below, neutron sources have been regularly used to study residual strains in thermally sprayed and cold sprayed coatings. For coated samples 


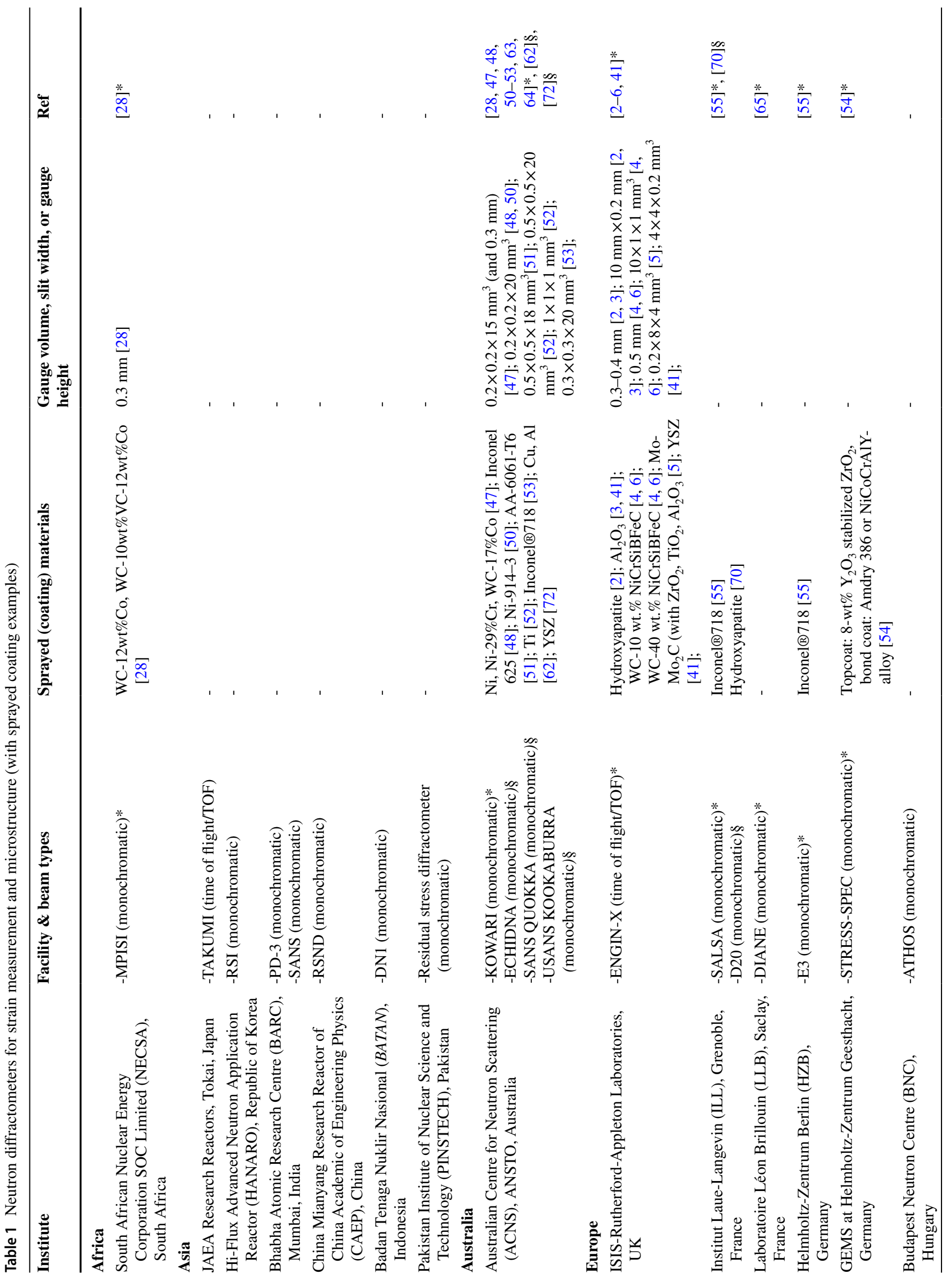




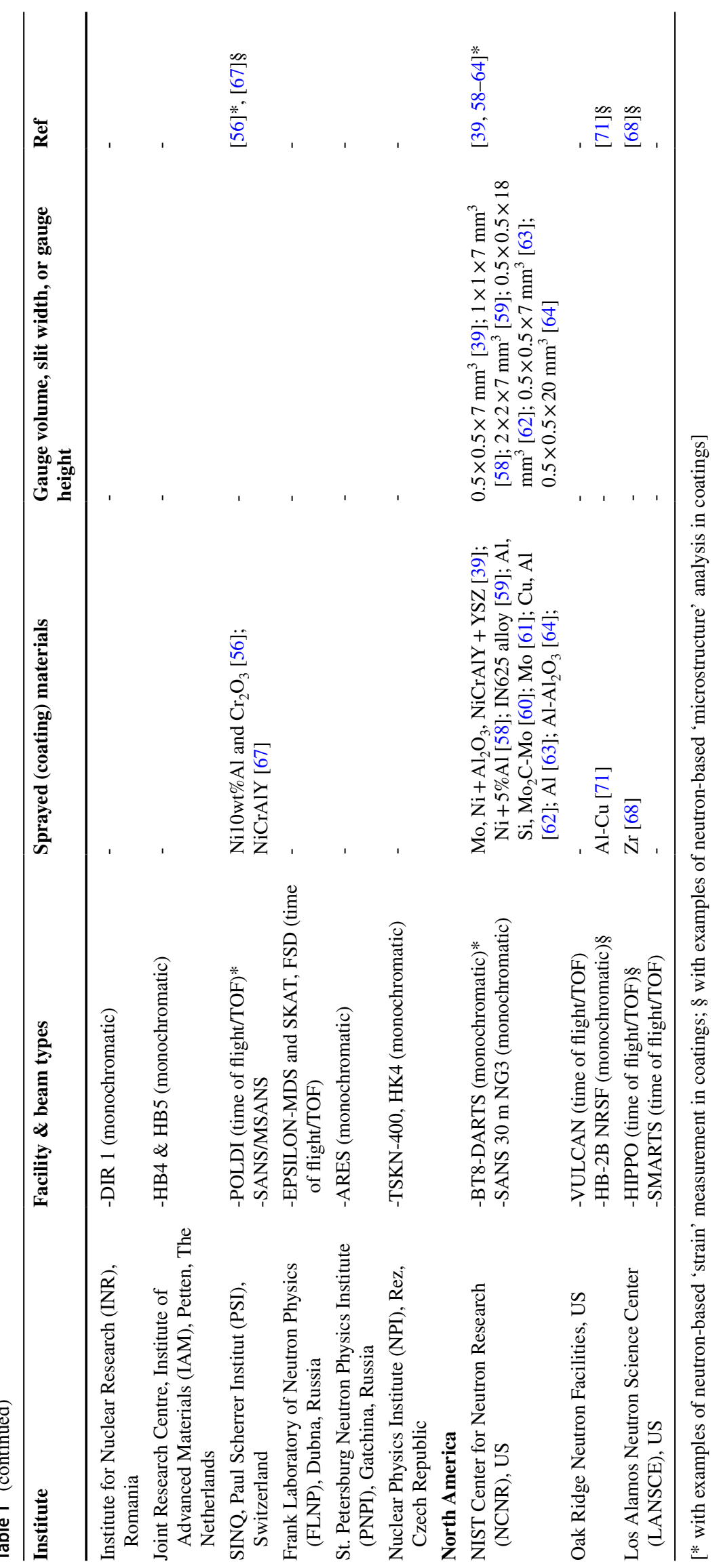


of various thicknesses, investigators have used various gauge volumes with slit widths $(0.2 \mathrm{~mm}, 0.3 \mathrm{~mm}, 0.5 \mathrm{~mm})$ in through thickness dimension. Interestingly, different strategies were considered to obtain strain-free lattice parameter $\left(d_{h k l}^{0}\right)$ for the coating and substrate part. However, to measure residual strains (and stresses) averaged over gauge volume, $d_{h k l}$ (in-plane component) and $d_{h k l}$ (normal-plane component) values were used mainly, using an equal-biaxial stress approach, and implementation of elastic constant (Hooke's law).

Using KOWARI instrument at ANSTO, Venter et al. [28] studied $200 \mu \mathrm{m}$ thick HVOF sprayed coatings (e.g., $\mathrm{WC}-12 \mathrm{wt} \% \mathrm{Co}$, WC-10wt\%VC-12wt \%Co) deposited on to $4 \mathrm{~mm}$ thick mild steel substrate. The residual strains were investigated in the erosion wear scar regions. While using the test protocols of MPISI instrument [45], the focus was to study the strain behaviours in the substrates by employing through-thickness fine measurement, and by doing so, the stress in the coating could be determined by imposing strain balance in the sample. While using the test protocols of KOWARI instrument [46], with diffracted beam slit at a width of $0.3 \mathrm{~mm}$ and positioning the gauge volume in the centre of the $200 \mu \mathrm{m}$ thick coating, the strains were measured directly in the coatings [28]. It was proposed that it is almost impossible to provide unstressed reference material (due to thin layer of coatings) and used equal-biaxial stress approach. Overall, the neutron scattering results indicated that the erosion impact angles influenced the residual strains and the coating composition.

Using KOWARI instrument at ANSTO, Luzin et al. [47] studied through thickness residual stresses in three coating materials sprayed using HVOF (JP 5000, DJ2700). The samples included: (a) $0.5 \mathrm{~mm}$ thick Ni coating on AISI 1008 carbon steel substrate (2.6 mm thick plate) was deposited using JP 5000 (Praxair TAFA), (b) $0.25 \mathrm{~mm}$ thick Ni-29\% Cr coating on low carbon steel substrate (1.64 $\mathrm{mm}$ thick plate) was deposited using DJ2700 (Diamond Jet gun) and (c) $0.25 \mathrm{~mm}$ thick WC-17\%Co coating on ferritic and austenitic steel substrates ( $2.2 \mathrm{~mm}$ thick plate) was deposited using JP 5000 (Praxair TAFA). A gauge volume $0.2 \times 0.2 \times 15 \mathrm{~mm}^{3}$ (as well as $0.3 \mathrm{~mm}$ ) in the through thickness dimension was used and applied equal-biaxial stress approach to obtain residual stresses. In the examples shown, it was observed that the method was capable for stress characterisation in coatings with thickness ranging from $0.2 \mathrm{~mm}$ to $0.5 \mathrm{~mm}$, and through-thickness resolution of $0.2 \mathrm{~mm}$ can be useful for routine and serial measurements in various coating substrate systems, potentially enabling optimisation of spray process parameters.

Using KOWARI instrument at ANSTO, Oladijo et al. [48, 49] studied through thickness residual stresses in HVOF sprayed $250 \mu \mathrm{m}$ to $500 \mu \mathrm{m}$ thick Inconel 625 coating on 304 stainless steel substrates of $3 \mathrm{~mm}$ thickness. A gauge volume $0.2 \times 0.2 \times 20 \mathrm{~mm}^{3}$ in the through thickness dimension was used and applied equal-biaxial stress approach to obtain residual stresses. It was observed that the residual stressed were compressive in nature (approximately same for all samples), and the residual stresses increased slightly with increased coating thickness.

Using KOWARI instrument at ANSTO, Smith et al. [50] studied through thickness residual stresses in HVOF sprayed $365 \mu \mathrm{m}$ thick Ni-914-3 deposited on two different substrate geometries. The two geometries were cylindrical hourglass (cold-drawn 1018 steel, $12.7 \mathrm{~mm}$ diameter and $101.6 \mathrm{~mm}$ length), and flat beams (machined and heattreated 1008 mild-steel, $228.6 \times 25.4 \times 2.38 \mathrm{~mm}$ ). Residual strain measurements were performed with a gauge volume of $0.2 \times 0.2 \times 20 \mathrm{~mm}^{3}$, with the elongated gauge volume (oriented parallel to the in-plane direction and crack interface). Interestingly, stresses were measured in the three principal directions (i.e., two in-plane and one normal to the coating surface). Residual strain in substrate only (i.e., without coating) was also measured using the same method to address possible pre-coating stress within the substrate. The stress profiles were then subtracted from the stress profiles of the coated samples so that the reported stress profiles were associated with the spraying process only. Two methods for the residual stress measurement were used (i.e., before and after subjection of coated samples to a partial loading regime via cantilever bend fatigue). It was observed that the distribution of the residual stresses within a coating-substrate system can be influenced by the application of external mechanical loading stresses into the system. The effect of increased compressive residual stress within the coating surface during cantilever fatigue loading was also observed, due to the geometry and test configurations.

Using KOWARI instrument at ANSTO, Saleh, Luzin and Spencer [51] studied through thickness residual stresses in two coating materials sprayed using cold spray techniques (i.e., kinetic metallization or low-pressure cold spray, and supersonic cold spray). The samples included 3-4 mm thick AA-6061-T6 cold spray coatings on Al and Mg substrates (each $3 \mathrm{~mm}$ thick). Residual strain measurements were performed with a gauge volume of $0.5 \times 0.5 \times 18 \mathrm{~mm}^{3}$. Stresses were measured in the two principal directions (i.e., in-plane and one normal to the coating surface) and applied equalbiaxial stress approach to obtain residual stresses. Residual strain in substrate only (i.e., without coating) was also measured using the same method to address possible pre-coating stress within the substrate. The stress profiles were then subtracted from the stress profiles of the coated samples so that the reported stress profiles were associated with the spraying process only. It was observed that the $\mathrm{Al}$ coating is in slight compression, and regardless of the substrate material, the two $\mathrm{Al}$ coating stress profiles were similar, because of similarity in thermal and mechanical properties of $\mathrm{Al}$ and $\mathrm{Mg}$. 
Using KOWARI instrument at ANSTO, Luzin et al. [52] studied through thickness residual stresses in cold sprayed additively manufactured (CSAM), thick patch coated sample produced from titanium powder. The coating of thickness $3.2 \mathrm{~mm}$ was deposited on $6.2 \mathrm{~mm}$ thick stainless-steel substrate of size $30 \mathrm{~mm} \times 30 \mathrm{~mm}$, whereas the long bar of crossSect. $5.2 \mathrm{~mm}$ thickness and $3.44 \mathrm{~mm}$ width was sprayed on Al substrate $(66 \mathrm{~mm} \times 66 \mathrm{~mm} 2.8 \mathrm{~mm})$. For coating sample, residual strain measurements were performed with a gauge volume of $0.5 \times 0.5 \times 20 \mathrm{~mm}^{3}$. Stresses in coating were measured in the three principal directions (i.e., two inplane and one normal to the coating surface). Residual strain in substrate only (i.e., without coating) was also measured using the same method to address possible pre-coating stress within the substrate. The stress profiles were then subtracted from the stress profiles of the coated samples so that the reported stress profiles were associated with the spraying process only. For bar sample, residual strain measurements were performed with a gauge volume of $1 \times 1 \times 1 \mathrm{~mm}^{3}$. The three principal stress components can be calculated using the measurements taken from the three principal directions with the constant $d_{h k l}^{0}$. Isotropic elastic diffraction constants were used to calculate the stresses from the measured strains. From analysis, it was observed that in both sample types, the residual stress was mainly formed through thermal mismatch mechanism, due to the coefficient of thermal expansion (CTE) difference between substrate and deposited coating. The overall results also demonstrate the dependence of the stress state on the sample geometry. A change in the sample geometry led to the stress being measured change from a biaxial state to a uniaxial state.

Using KOWARI instrument at ANSTO, Kim et al. [53] studied the effect of low temperature range heat treatments $\left(100-400{ }^{\circ} \mathrm{C}\right)$ on the residual stress of cold sprayed Inconel@718 coating of 1.1-1.4 mm thickness deposited onto a pre-solution-treated A17075-T651 substrate (305 mm $\times 305 \mathrm{~mm} \times 24 \mathrm{~mm}$ ). Residual strain measurements were performed with a gauge volume of $0.3 \times 0.3 \times 20 \mathrm{~mm}^{3}$. Stresses were measured in the two principal directions (i.e., one in-plane and one normal to the coating surface). The coating was assumed to be in a bi-axial stress state with the stress in the direction normal to the surface assumed to be zero for thin coatings. Kim et al. [53] suggested that the existence of zero stress condition in the direction normal to the thickness is a valid assumption for the coating with the thickness of up to $2 \mathrm{~mm}$ but not for the substrate with much higher thickness. This assumption is still valid for the substrate till a distance of $2 \mathrm{~mm}$ from the interface. The effect of the heat treatment temperature on the stress relaxation was also demonstrated. Lower temperature helped relieve the stresses, whereas a higher temperature resulted in a residual compressive stress in the Inconel 718 coating and a residual tensile stress in the substrate.
Using the test protocols of STRESS-SPEC@FRM II instrument at GEMS, Gibmeier et al. [54] studied local phase and residual strains for yttria stabilised zirconia (YSZ) thermal barrier coatings (TBC). The coatings were deposited using a mix of techniques (air plasma spray/APS; high velocity oxy-fuel/HVOF; vacuum plasma spray/VPS) on two different substrates (Inconel IN 738 LC, nickel-based superalloy/Mar M 247; $32 \mathrm{~mm}$ diameter and $3 \mathrm{~mm}$ thick). After coating, the samples were subjected to cyclic and isothermal heat treatments. Through surface strain scanning was carried for local residual stress analysis to analyse diffraction data from all contributing layers (i.e., topcoat: 8-wt $\% \mathrm{Y}_{2} \mathrm{O}_{3}$ stabilized $\mathrm{ZrO}_{2}$ with thicknesses $360 \mu \mathrm{m}$ to $671 \mu \mathrm{m}$; bond coat: Amdry 386 or NiCoCrAlY-alloy with thicknesses $205 \mu \mathrm{m}$ to $230 \mu \mathrm{m}$ ) and of the substrate material. However, to measure residual strains, $d_{h k l}$ (heat-treated) was compared to $d_{h k l}^{0}$ (as sprayed). It was proposed that it is almost impossible to provide unstressed reference material (due to thin layer of coatings) with identical microstructure to determine stressfree $d_{h k l}^{0}$. From the measured strain, the residual stresses were determined using the elastic constant (Hooke's law) for material types determined based on bending experiments. The heat treatment led to the YSZ topcoat exhibiting a compressive residual stress in the in-plane and out-of-plane direction. Whereas, in the bond coat a tensile residual stress was seen for the reason of balancing the stress. Compared to isothermal loading, cycling thermal loading led to a higher degree of compressive residual stresses towards the surface of the YSZ topcoat. Similarly, the degree of tensile loading within the bond coat increased with cyclic thermal loading.

Using an HMI instrument (currently HZB) and SALSA instrument at ILL, Lyphout et al. [55] investigated residual strains in HVOF sprayed Inconel 718 coatings (four different thicknesses: 1, 1.5, 2, $2.5 \mathrm{~mm}$ ) on Inconel 718 substrates (25.4 $\mathrm{mm}$ diameter and $3 \mathrm{~mm}$ thickness). In their experiments, a freestanding coating, and an annealed grit blasted substrate respectively to balance the stress to obtain the $d_{h k l}^{0}$ value. Reflection and transmission modes were used on the sample from top to bottom to calculate the radial and axial stresses. From the measured strain, the residual stresses were determined using the elastic constant (Hooke's law) for material types. Increasing the coating thickness (i.e., $1 \mathrm{~mm}$ to $2.5 \mathrm{~mm}$ ) did not modify through-thickness residual stress in coatings, and overall compressive stress was achieved both in coating and substrate at the interface, but the difference in stress amplitude at the interface seems to significantly decrease when coating thickness was increased.

Using POLDI instrument at SINQ, Kovářík et al. [56] investigated plasma sprayed Ni10wt\% Al (about $100 \mu \mathrm{m}$ thick) and $\mathrm{Cr}_{2} \mathrm{O}_{3}$ (about $150 \mu \mathrm{m}$ thick) onto mild steel (S235JRC, $4 \mathrm{~mm}$ thickness) substrate. Through thickness residual strain profile (slightly below the substrate surface) was measured in the mild steel substrate only due to very 
low diffracted signal in coatings part. Strain-free lattice spacing $\left(d_{h k l}^{0}\right)$ was determined from the measurement at the centre of the coated specimen. From the measured strain, the residual stresses were determined using the elastic constant (Hooke's law) for material types (analysed by selecting the most intense peak of the spectra). In the example shown, it was observed that the compressive residual stress (originated by grit blasting and preserved below the $\mathrm{Cr}_{2} \mathrm{O}_{3}$ layer) was present close to the substrate surface, and the maximum value of compressive stress was measured in the coating combination (i.e., $\mathrm{Ni} 10 \mathrm{wt} \% \mathrm{Al}$ on $\mathrm{Cr}_{2} \mathrm{O}_{3}$ specimen).

Some of the pioneering work related to residual strain analysis in thermal spray coatings were carried at NIST (e.g., Kesler et al. [39]; Gnäupel-Herold et al. [57]; Matějíček et al. [58]; Gnäupel-Herold et al. [59]) where distinguished stresses in the different phases of a coating and throughthickness stress profile for thick coating as well as average value for thin coating were presented. Technique to measure residual stress in thermal spray coatings using BT8 instrument at NIST is explained in detail by Keslar et al. [39], where two distinct material classes were investigated. The coating materials included Mo which was a single-material coatings and $\mathrm{Ni}+\mathrm{Al}_{2} \mathrm{O}_{3}$ and $\mathrm{NiCrAlY}+\mathrm{YSZ}$ bi-material composites, with and without graded layers. For neutron diffraction measurements, $2 \mathrm{~mm}$ thick coatings deposited by APS technique on $2.5 \mathrm{~mm}$ thick steel substrates were used. Keslar et al. [39] used a gauge volume of $5 \times 5 \times 5 \mathrm{~mm}^{3}$ centred over the material of interest (i.e., coating, substrate) to eliminate the partial illumination effects, obtaining average stress values from the entire coating thickness. However, when the stress gradient through the coating thickness was of interest and when the coating had sufficient thickness, the gauge volume was reduced to $0.5 \times 0.5 \times 7 \mathrm{~mm}^{3}$, obtaining a through-thickness stress profile. Further on, Matějíček et al. [58] compared through thickness residual stress in $\mathrm{Ni}+5 \% \mathrm{Al}$ coatings sprayed using two techniques (APS: $1.9 \mathrm{~mm}$ thick coating on $2.5 \mathrm{~mm}$ thick steel substrate, cold spray: $1.8 \mathrm{~mm}$ thick coating on $2.9 \mathrm{~mm}$ thick steel substrate) using the gauge volume of $1 \times 1 \times 7 \mathrm{~mm}^{3}$. In the APS coating, the average residual stress was tensile, with a positive gradient because of consecutive deposition of layers with tensile quenching stress. It was observed that due to similar coefficient of thermal expansion of the coating and substrate materials and relatively low deposition temperature the thermal stress contribution was small. However, in the coldsprayed coating, the average stress was compressive, due to high-velocity of sprayed particle impact that causes plastic deformation in the underlying layers and thus compressive stress (i.e., peening effect). In a novel work, Gnäupel-Herold et al. [59] investigated the effect of feedstock particle size (classed as fine, medium, coarse, mixed) and coating porosity (fine: $1.5 \%$, coarse: $4.6 \%$ ) on the residual stress of plasma sprayed Ni-Cr-Mo-Fe-Mb-Ti-Al (or IN625 alloy) coating of thickness $0.4-0.7 \mathrm{~mm}$ deposited on low carbon steel substrate of $2.9 \mathrm{~mm}$ thickness, using the gauge volume of $2 \times 2 \times 7 \mathrm{~mm}^{3}$. It was observed that the residual stress level declined strongly with the increase in the particle size and porosity. Gnäupel-Herold et al. [59] also suggested that the quenching strains are assumed to be equal, thus indicating that the Young's modulus largely controls the residual stresses in the coatings.

Using the BT8 instrument at NIST, Luzin et al. [60] investigated various thermally sprayed coatings ranging from thick deposits (about $10-30 \mathrm{~mm}$ thick) to less than $500 \mu \mathrm{m}$ thick coatings. The coatings included cold sprayed $\mathrm{Al}$ powder on $\mathrm{Al}$ substrate, plasma sprayed $\mathrm{Si}$ powder on $\mathrm{Cu}$ substrate, $\mathrm{HVOF}$ and plasma sprayed $\mathrm{Mo}_{2} \mathrm{C}$-Mo powder on steel substrate and iron sample by spray forming. They implemented different residual stress measurement strategy for coatings of different thicknesses. For example, for thin coatings, $(<0.5 \mathrm{~mm})$, an average through thickness stress value was calculated along with a normal and in-plane stress to resolve the in-plane stress and the stress-free d-spacing $\left(d_{h k l}^{0}\right)$. For medium thickness coating $(0.5<\mathrm{t}<5 \mathrm{~mm})$, the assumption of zero normal stress was approximated, however, the through-thickness measurement was possible, including two measurements per measurement point were suggested to be sufficient. For thick coating ( $\mathrm{t}>5 \mathrm{~mm}$ ), the assumption of normal stress (to be zero) was suggested. For thick coating, to resolve the three principal stress components, the knowledge of at least three independent measurements and the stress free spacing $\left(d_{h k l}^{0}\right)$ is required. It was proposed that owing to their high penetration, neutrons allow stress profiling through the coating thickness, and multiphase materials can be studied, including the effect of other surface treatments, such as shot peening, and laser peening.

Using BT8 instrument at NIST, Li et al. [61] investigated stress magnitude of both splats and coatings on substrates (using X-ray micro-diffraction) as well as entire coating thickness stress (using neutron diffraction) for the $380 \mu \mathrm{m}$ thick coatings of plasma sprayed molybdenum deposited onto 1040 steel ( $2.4 \mathrm{~mm}$ thick). From the measured strain, the residual stresses were determined using the elastic constant (Hooke's law) for material types (analysed by selecting the most intense peak of the spectra). Though no apparent residual stress was found in the $380 \mu \mathrm{m}$ coating, a single splat layer exhibited a high residual stress due to the high degree of bonding of the first splat layer and stress relaxation within the coating due to the large number of defects.

Using the BT8 instrument at NIST, Luzin et al. [62] in continuation of Choi et al. [63] work related to cold spray $\mathrm{Al}$ coatings, investigated surface and in-plane stresses in $\mathrm{Cu}$ and $\mathrm{Al}$ cold sprayed coatings. Cold sprayed $\mathrm{Cu}$ (onto $\mathrm{Cu}$ and $\mathrm{Al}$ substrates) and $\mathrm{Al}$ (onto $\mathrm{Cu}$ and $\mathrm{Al}$ substrates) samples were about $3 \mathrm{~mm}$ thick. The $\mathrm{Cu}$ substrates were $3.1 \mathrm{~mm}$ thick, whereas the $\mathrm{Al}$ substrates were $2.6 \mathrm{~mm}$ thick [62]. 
A gauge volume $0.5 \times 0.5 \times 18 \mathrm{~mm}^{3}$ was used. The internal stress in the free-standing substrates were measured before the cold spraying, and this internal stress was subtracted to isolate stresses induced by the cold spray process. Additionally, Luzin et al. [62] investigated a $\mathrm{Cu}$ coating onto Al substrate at KOWARI instrument at ANSTO while maintaining the measurement protocol as close possible (i.e., no practical difference and using appropriate elastic constants but selecting $\mathrm{Cu}$ (222) reflection for analysis). From the measured strain, the residual stresses were determined using the assumption of a balanced biaxial plane stress state [63]. It was observed that the kinetic parameters of the cold spray process, the deformation behaviour of the particles and the feedstock material properties dictate the residual stress within the coating. Compared to $\mathrm{Al}$ coatings, the mechanical properties of the $\mathrm{Cu}$ coating were closer to bulk properties. The higher plastic strain the $\mathrm{Cu}$ coatings resulted in higher residual stress in the $\mathrm{Cu}$ coatings and higher compaction [62].

Using the BT8 instrument at NIST, Luzin et al. [64] investigated residual stresses in low-pressure cold sprayed coatings (e.g., $\mathrm{Al}-\mathrm{Al}_{2} \mathrm{O}_{3}$ metal matrix composite) of $1 \mathrm{~mm}$ thickness onto $3 \mathrm{~mm}$ thick A16061 aluminium substrate. A gauge volume $0.5 \times 0.5 \times 20 \mathrm{~mm}^{3}$ was used and applied equal-biaxial stress approach to obtain residual stresses. In the example shown, it was observed that the overall residual stress in the coating was compressive. Also, due to the elevated spray temperature characteristic of low-pressure cold sprayed coatings (dynamic metallization), thermal stresses were also present, and because of the multi-phase composition and thermal mismatch between the metal and ceramic components of the metal matrix composite (MMCs), interphase micro-stresses also accumulated, leading to overall compressive residual stresses [64].

Not much information available, but using DIANE instrument at LLB, Ceretti et al. [65] investigated residual strain profile from the coating surface to the material through the interface in a ferritic steel cylinder having $78 \mathrm{~mm}$ diameter. The elastic constant (Hooke's law) related to the most intense peak of the spectra were measured with respect to the three principal directions.

\section{Other Analysis}

Small-angle neutron scattering (SANS) techniques [66] helps analyse the total specific surface area of the voids (size up to about $100 \mathrm{~nm}$ ), whereas multiple small-angle neutron scattering (MSANS) technique can help analyse average pore size and orientational distribution.

Using SANS and MSANS techniques likely carried at PSI (as diffraction source was not clearly stated), Keller et al. [67] studied the void microstructures (i.e., interlamellar pores, intra-lamellar cracks, and volumetric globular voids, each having different volume fractions, anisotropy, size, and shape) of APS sprayed NiCrAlY coatings. Keller et al. [67] suggested that for the NiCrAlY coating, inter-lamellar cracks constituted only a small portion of the void fraction, whereas the interlamellar pores were a major portion of the void system.

The HIPPO instrument at LANSCE provides a range of material information including crystallographic textures, phase fractions, lattice parameters, and micro-strain. At HIPPO instrument, Takajo et al. [68] investigated crystallographic textures and phase fractions of involved phases of $23.8 \mu \mathrm{m}$ to $34.0 \mu \mathrm{m}$ thick $\mathrm{Zr}$-coatings deposited on $\mathrm{U}-10 \mathrm{Mo}$ foils $(0.3 \mathrm{~mm}$ thick). Texture, being responsible for the anisotropic physical properties of materials, reflects the thermomechanical history including recrystallisation, particle rotation and phase transformation. In addition, the bulk texture of a polycrystalline material constitutes the orientation distribution function of the crystallographic orientation with respect to the sample coordinate system [69]. The texture analysis of the coated surface showed [68] that selective grain growth likely caused considerable change in the texture at high temperatures. Also, Takajo et al. [68] suggested that $\mathrm{Zr}$ coating showed a preferential orientation and correlated with the initial texture of the uncoated U-10Mo.

Using D20 instrument at ILL, Jakani et al. [70] investigated crystallinity of plasma sprayed hydroxyapatite coatings (feedstock was micro and nano-sized particles) deposited onto a Ti-6Al-4 V substrate. The sample was heat-treated $\left(2 \mathrm{~h}\right.$ at $\left.600{ }^{\circ} \mathrm{C}\right)$ to reduce amorphous fractions. Using the neutron spectra of samples (feedstock powder and coatings), Jakani et al. [70] observed that the deposited coating showed retention of the nano-meter crystallite and no secondary phases were identified. This meant that the nanocoating will have nucleation sites to the reconstituted bone, which will allow stabilisation of the implant in the bone.

Using HB-2B instrument at ORNL, Liu et al. [71] investigated the plastic deformation in a series of low-pressure cold sprayed Al-Cu coatings (on Al-Cu-Mg-Mn alloy (AA2024) substrate) using dislocation density as an indicator. For the copper content ranged from 2 mass $\%$ to 5 mass\%. Analysing the neutron diffraction peaks, it was observed that the increase in the copper alloy additions (from 2 mass\% to 5 mass\%) increased the breadths of full width at half maximum (FWHM) of the cold sprayed coatings increased, meaning the dislocation density in the Al-Cu coatings systematically increased with increasing $\mathrm{Cu}$ alloy content. Liu et al. [71] also showed that the peak breadth (i.e., FWHM) changes from the substrate to the coating and through the coating, and this change throughout the coating was accounted for by the fact that the regions near the coating-substrate interface saw a larger number of repetitive impacts from subsequent 
particles than the rest of the areas towards the top surface of the coatings.

Using the powder diffractometer ECHIDNA at ANSTO OPAL (wavelength of $1.622 \mathrm{~A}^{\circ}$ ), Luzin et al. [62] measured oxide volume fraction of the cold sprayed coatings and compared against the feedstock powder (i.e., $\mathrm{Cu}, \mathrm{Al}$ ), including analysis to confirm the chemical purity of the feedstock powders. It was experienced that the usage of neutron powder diffractometer is better compared to X-ray diffraction, because the X-ray diffraction could only provide surface properties and not the subsurface, whereas the neutron diffractometer provides a better average measurement.

Recently, Tejero-Martin et al. [72] used neutron scattering to study the pore size distribution and total porosity in thermally sprayed coatings were performed at ANSTO OPAL using SANS QUOKKA instrument (wavelength of $5 \mathrm{~A}^{\circ}$ and $8.1 \mathrm{~A}^{\circ}$ ) and using ultra-small angle or USANS KOOKABURRA instrument (wavelength of $2.37 \mathrm{~A}^{\circ}$ ). They investigated pore size distribution and total porosity of freestanding $150 \mu \mathrm{m}$ thick heat-treated suspension high-velocity oxy fuel (S-HVOF) YSZ thermal barrier coatings. Analysis indicated that such combination of SANS and USANS can study porosity of radii between $1 \mathrm{~nm}$ and $10 \mu \mathrm{m}$ and can easily differentiate the as-sprayed coatings with heat-treated coatings.

\section{General Assessment of Examples}

As demonstrated in previous sections (i.e., test preparation, test set-up, and analysis), neutron diffraction is usually very time-consuming process to produce spatially resolved strains, as it requires scanning, measuring one point after the other in through thickness strain mapping of coated sample. Also, through examples summarised above, it appears that the evaluation of residual strain is of interest across many neutron scattering facilities worldwide for the characterisation of thermally and cold sprayed coatings. Table 1 summarise examples where sprayed coatings have been characterised to study strains as well as microstructures using neutron diffraction techniques.

Since most neutron sources operate dedicated neutron strain scanners, it appears coherent that a lot of the pioneering work from both or either of beam types (i.e., monochromatic and time of flight/TOF) have been performed at these facilities. While in TOF measurements the wavelength coverage is less of a concern and only measurements of a certain wavelength range are efficient, in the case of monochromatic measurements it comes at the cost of much added measurement time [73]. To carry further assessment with neutron diffracted lattice strain data, it is expected that this capability can be especially useful for the optimisation of new coating processing techniques with implications to an improved coating-substrate structural integrity. However, for microstructure analysis (e.g., porosities, void morphologies, texture), this technique could become very time consuming for the study of inhomogeneous coated layer with spatially altered texture and phase distribution.

As discussed for residual strain cases, there are several examples above showing the strain measurement on thinner coatings (in 100's of micrometres) as well as thicker coatings (in few millimetres) with dependencies on sub-millimetre or smaller gauge volume for coating parts and obtaining strainfree lattice parameter $\left(d_{h k l}^{0}\right)$ for the coating and substrate part. The number of testing in coating-substrate system is growing worldwide and pushing the boundaries in resolution, measurement, and instrument optimisation and particular in microstructural analyses and lattice strain quantification.

The benefits of neutron diffraction to study the effect of mechanical loading on thermally sprayed coatings are obvious, for example Smith et al. [50] studied through thickness residual stresses in samples before and after subjection of coated samples to a partial fatigue loading regime, influencing the sample properties. Usage of existing as well as development of new environmental test chamber will continue across neutron diffraction facilities, however, there has been very little or no studies where samples has been tested at range of temperatures or loading conditions. It is expected that experiments in environmental test chamber could become routine for the users at neutron facilities.

While not much information is available, but there are some good examples of institutional collaborative studies where neutron diffraction (mainly with monochromatic beam type) complimentary and/or comparative analysis of thermally sprayed coating has been carried on sprayed coated samples. For example, KOWARI instrument at ANSTO in partnership with MPISI instrument at NECSA [74] were used to investigate residual stresses in the wear scar regions of coated sample. The test at MPISI instrument focused on the stress behaviours in the substrates by employing a fine measurement through-thickness, from which the coating stress could be determined by imposing stress balance in the coating-substrate system. The measurement at MPISI instrument (called as indirect approach) required sample alignment and many through-thickness data points to compensate for the unfavourable thickness ratio between the coating $(0.2 \mathrm{~mm})$ and the substrate $(4 \mathrm{~mm})$. While the test at KOWARI instrument (called as direct approach) focused on stress measurement directly in the coatings, where prolonged measurement times were applied to accumulate adequate diffracted intensity from the thin coating for the calculation of stresses. However, both measured results indicated that the erosion impact angles have an influence on the residual stresses and the coating composition. In another example, partnership with HMI instrument (currently HZB) and SALSA instrument at ILL [55] allowed analyses of only stress profiles in the substrate, as diffraction angle close to 
$90^{\circ}$ gave high spatial resolution, but not enough signal intensity to measure the broader peaks of the coating. Therefore, to gain in response intensity, the coatings most intense peak was considered at SALSA, leading to full coating-substrate system analysis for residual stresses. However, issues related to interface measurements led to overestimation (and uncertainty) of local compressive stresses, due to large sampling volume. It was proposed that a deconvolution procedure (i.e., modelling the Gaussian-like distribution of the intensity through the gauge volume may increase the resolution in the interface region). In another example, BT8 instrument at NIST in partnership with KOWARI instrument at ANSTO [64] studied residual stress using BT8 instrument and texture using KOWARI instrument. Such collaboration is good for informed test measurement and guidance along with potential to open new avenue of future research.

Neutron diffraction techniques complement other characterisation techniques and number of neutron facilities worldwide are considered the most useful for materials of today as well for future new materials. Combination of diffraction techniques as well as advances in sample environments and neutron sources will make such experiments more routine for the users at neutron facilities, which can be applied to coating materials as well. Meanwhile, some of the trends which we can expect to continue are advances in neutron target stations, diffractometers (detectors), instrumentation for faster acquisition times, new environmental chambers to study coating materials under extreme conditions, new coating-substrate materials and applications, individual splat level analysis, improved coating-substrate interface residual strain data, along with spatially resolved distribution of the coating properties of interest.

\section{Concluding Remarks}

In terms of residual strain measurement advancement, it has been argued that X-ray synchrotron (diffraction) nondestructive methods results in radiation that does not penetrate very well into the material subsurface layers and the substrate. However, in recent times neutron diffraction technique has been used on numerous thermal (or cold) spray coating materials to measure residual strains including microstructures. Likewise, neutron diffraction has been argued to be superior (but expensive and potentially timeconsuming) for characterisation purposes especially in the characterisation of the structural evolution during heating, annealing, and cooling because of deeper penetration of the neutron beam, though at lower flux compared to laboratory or synchrotron X-ray sources.

While applying neutron diffraction test protocols and implementing appropriate analysis strategies, from examples discussed in sections above, there are several studies where residual strains have been investigated for materials deposited through thermal or cold spray methods at neutron diffractometers mainly at ISIS/ENGIN-X, ANSTO/KOWARI and NIST/BT8, and some at NECSA/MPISI, ILL/SALSA, LLB/DIANE, HZB/E3, GEMS/STRESS-SPEC, and PSI/ POLDI. Apart from residual strain analysis, microstructures of thermal or cold spray coatings have also been investigated at other neutron diffractometers (e.g., BARC/SANS, ANSTO/(ECHIDNA, QUOKKA, KOOKABURRA), ILL/ D20, NIST/SANS, ORNL/HB-2B, and LANSCE/HIPPO). Overall, for coated samples of various thicknesses, investigators have used various gauge volumes with slit widths (e.g., $0.2 \mathrm{~mm}, 0.3 \mathrm{~mm}, 0.5 \mathrm{~mm}$ ) in through thickness dimension. Interestingly, different strategies were considered to obtain strain-free lattice parameter $\left(d_{h k l}^{0}\right)$ for the coating and substrate part, and to measure residual strains (and stresses) averaged over gauge volume, $d_{h k l}$ (in-plane component) and $d_{h k l}$ (normal-plane component) values were used mainly, using an equal-biaxial stress approach, and implementation of elastic constant (Hooke's law).

Neutron diffraction using time-of-flight diffractometers is particularly useful at the coating-substrate interface as when the gauge volume is half/half in the coating-substrate system, one can identify both materials properties without losing any positioning accuracy in terms of the centre of gravity of the neutron beam. Additionally, single and multiphase materials can be successfully investigated using either monochromatic $[39,75]$ or time-of-flight sources.

Research in thermal spray area, however, does require further comparatively evaluation of destructive, nondestructive techniques or combination of both, to understand the through thickness residual stress within the coating-surface system. The cross correlation of the independent techniques is vital, as measured values of stress in the coating-substrate system can be sensitive to the stress measurement technique, which in turn influences the validation of the numerical and mathematical methods of residual stress measurement [16] and monitoring in such systems.

Non-destructive measurement of these strain (or stress) fields in relatively thin thermal spray coatings also poses a challenge, as the techniques such as laboratory X-ray and synchrotron X-ray are capable of only providing a subsurface strain measurement. Surface roughness effects further complicate the very near-surface residual stress measurements which are important for near-surface crack initiation during wear. This drawback poses a challenge with residual stress measurement in thermal spray coatings as the through thickness stress measurement would require layer removal. There is also very limited literature that discuss correlations between strain measurement, structure-property relations, and effect of post-treatment on the performance of such coatings which can result in harmonization of the 
strain field within the coating, and at the coating-substrate interface.

Other emerging challenges include testing new composite coating materials and new coating techniques applied (e.g., suspension or solution based thermal spray coatings manufacturing using nanostructured feedstock powders [e.g., 41, 72]), including strain testing on actual coated parts of various geometries (e.g., curved, spherical, cylindrical, edges, corners, etc.). While the small grain size is the limiting factor for the applicability of neutron diffraction, there are other approaches and schemes for testing and predicting residual stresses in thermal spray coatings [76], however, issues regarding the accuracy, applicability, distribution analysis can be further investigated using principles of artificial intelligence (AI) and machine learning (ML) approaches [77, 78].

\section{Appendix A. Supplementary data}

Experimental raw data (example) associated with this article can be found online at the data journal; https://data.isis.stfc. ac.uk/doi/INVESTIGATION/58451518/.

Acknowledgements The authors and instrument scientists acknowledge gaining neutron strain testing and analysis experience through several awards of ENGIN-X beamtime at the STFC ISIS facility for the neutron diffraction measurements of thermal spray coatings (e.g., RB1510238, RB910205, RB810413, RB15141, RB1120507, RB13990, RB38308).

CRediT Authorship Contribution Statement Nadimul Faisal: Conceptualisation, Data curation, Formal analysis, Funding acquisition, Investigation, Methodology, Project administration, Resources, Software, Validation, Writing — original draft. Rehan Ahmed: Conceptualisation, Funding acquisition, Investigation, Methodology, Project administration, Resources, Validation, Writing—review \& editing. Anil Prathuru: Writing—review \& editing. Anna Paradowska: Methodology, Testing, Writing - review \& editing. Tung-Lik Lee: Methodology, Testing, Writing—review \& editing.

\section{Declarations}

Conflict of Interest The authors declare that they have no known competing financial interests or personal relationships that could have appeared to influence the work reported in this paper.

Open Access This article is licensed under a Creative Commons Attribution 4.0 International License, which permits use, sharing, adaptation, distribution and reproduction in any medium or format, as long as you give appropriate credit to the original author(s) and the source, provide a link to the Creative Commons licence, and indicate if changes were made. The images or other third party material in this article are included in the article's Creative Commons licence, unless indicated otherwise in a credit line to the material. If material is not included in the article's Creative Commons licence and your intended use is not permitted by statutory regulation or exceeds the permitted use, you will need to obtain permission directly from the copyright holder. To view a copy of this licence, visit http://creativecommons.org/licenses/by/4.0/.

\section{References}

1. Zhang SY, Evans A, Eren E, Chen B, Pavie M, Wang Y, Pierret S, Moat R, Mori B (2013) ENGIN-X - instrument for materials science and engineering research. Neutron News 24(3):22-26. https://doi.org/10.1080/10448632.2013.804362

2. Ahmed R, Faisal NH, Paradowska AM, Fitzpatrick M, Khor KA (2011) Neutron diffraction residual strain measurements in nanostructured hydroxyapatite coatings for orthopaedic implants. J Mech Behav Biomed Mater 4(8):2043-2054. https://doi.org/10. 1016/j.jmbbm.2011.07.003

3. Ahmed R, Faisal NH, Paradowska AM, Fitzpatrick M (2012) Residual strain and fracture response of $\mathrm{Al}_{2} \mathrm{O}_{3}$ coatings deposited via APS and HVOF techniques. J Therm Spray Technol 21:23-40. https://doi.org/10.1007/s11666-011-9680-7

4. Ahmed R, Fitzpatrick M, Faisal NH (2012) A comparison of neutron diffraction and hole-drilling residual strain measurements in thermally sprayed coatings. Surf Coat Technol 206:4180-4185. https://doi.org/10.1016/j.surfcoat.2012.04.018

5. Faisal NH, Ahmed R, Prathuru AK, Katikaneni SP, Goosen MFA, Zhang S-Y (2018) Neutron diffraction residual strain measurements of molybdenum carbide based solid oxide fuel cell anode layers with metal oxides on Hastelloy X. Exp Mech 58(4):585603. https://doi.org/10.1007/s11340-017-0298-7

6. Ahmed R, Yu H, Stoica V, Edwards L, Santisteban JR (2008) Neutron diffraction residual strain measurements in post-treated thermal spray cermet coatings. Mater Sci Eng, A 498:191-202. https://doi.org/10.1016/j.msea.2008.08.023

7. Wenzelburger M, López D, Gadow R (2006) Methods and application of residual stress analysis on thermally sprayed coatings and layer composites. Surf Coat Technol 201:1995-2001. https://doi. org/10.1016/j.surfcoat.2006.04.040

8. Development and applications of residual stress measurements using neutron beams. - Vienna: International Atomic Energy Agency, 2014. http://www-pub.iaea.org/MTCD/Publications/ PDF/trs477_web.pdf

9. Withers PJ, Bhadeshia HKDH (2001) Residual stress. Part 1 Measurement techniques. Materials Science and Technology 17(4):355-365. https://doi.org/10.1179/026708301101509980

10. Ahmed R, Hadfield M, Tobe S (1996) Residual stress analysis in thermal spray coated rolling elements. National Thermal Spray Conference, USA, ISBN 0-87170-583-4:875-883

11. Hadfield M, Ahmed R, Tobe S (1997) Residual stress measurements of silicon nitride and coated tungsten carbide rolling contact elements. Surface Treatment-III 17:319-328. https://doi.org/ 10.2495/SURF970311

12. Ahmed R, Hadfield M (1997) Experimental measurement of the residual stress field within thermally sprayed rolling elements. Wear 209:84-95. https://doi.org/10.1016/S0043-1648(97)00009-4

13. Hadfield M, Ahmed R, Tobe S (2000) Variation in residual stress fields during fatigue failure of thermal spray coatings. International Thermal Spray Conference, Canada, ISBN 0-87170-680-6:399-406

14. Stoica V, Ahmed R, Golshan M, Tobe S (2004) Sliding wear evaluation of hot isostatically pressed (HIPed) thermal spray cermet coatings. J Therm Spray Technol 13(1):93-107. https://doi.org/ 10.1007/s11666-004-0054-2

15. Fardan A, Ahmed R (2019) Modeling the evolution of residual stresses in thermally sprayed YSZ coating on stainless steel 
substrate. J Therm Spray Technol 28(4):717-736. https://doi.org/ 10.1007/s11666-019-00856-2

16. Fardan A, Berndt CC, Ahmed R (2021) Numerical modelling of particle impact and residual stresses in cold sprayed coatings: A review. Surf Coat Technol 409:126835. https://doi.org/10.1016/j. surfcoat.2021.126835

17. Matejícek J, Sampath S, Dubsky J (1998) X-ray residual stress measurement in metallic and ceramic plasma sprayed coatings. J Therm Spray Technol 7:489-496. https://doi.org/10.1361/105996398770350701

18. Bragg WH, Bragg WL (1913) The reflection of X-rays by crystals. Proceedings of the Royal Society 88(605):428-438. https://doi. org/10.1098/rspa.1913.0040

19. Allen AJ, Hutchings MT, Windsor CG, Andreani C (1985) Neutron diffraction methods for the study of residual stress fields. Adv Phys 34(4):445-473. https://doi.org/10.1080/00018738500101791

20. Price DL, Skold K (1986) Introduction to Neutron Scattering. Methods of Experimental Physics 23A:1-97. https://doi.org/10. 1016/S0076-695X(08)60554-2

21. Fitzpatrick ME, Lodini A (2003) Analysis of Residual Stress by Diffraction using Neutron and Synchrotron Radiation. Taylor and Francis, London

22. Schajer GS (2013) Practical Residual Stress Measurement Methods, John Wiley \& Sons Ltd, Online ISBN:9781118402832 (p. 1-27, p. 195-223). https://doi.org/10.1002/9781118402832

23. Hutchings MT, Withers PJ, Holden TM, Lorentzen T (2005) Introduction to the characterization of residual stress by neutron diffraction. CRC Press, Taylor and Francis, Boca Raton

24. Andersen KH et al (2020) The instrument suite of the European Spallation Source, Nuclear Instruments and Methods in Physics Research Section A: Accelerators, Spectrometers. Detectors and Associated Equipment 957:163402. https://doi.org/10.1016/j. nima.2020.163402

25. Santisteban JR, Daymond MR, James JA, Edwards L (2006) ENGIN-X: a third-generation neutron strain scanner. J Appl Crystallogr 39:812-825. https://doi.org/10.1107/S0021889806042245

26. Withers PJ, Preuss M, Steuwer A, Pang JWL (2007) Methods for obtaining the strain-free lattice parameter when using diffraction to determine residual stress. J Appl Crystallogr 40:891-904. https://doi.org/10.1107/S0021889807030269

27. James JA, Edwards L (2007) Application of robot kinematics methods to the simulation and control of neutron beam line positioning systems. Nucl Inst Methods Phys Res A 571:709-718. https://doi.org/10.1016/j.nima.2006.11.033

28. Venter AM, Luzin V, Marais D, Sacks N, Ogunmuyiwa EN, Shipway $\mathrm{PH}$ (2020) Interdependence of slurry erosion wear performance and residual stress in WC-12wt\% Co and WC-10wt\%VC-12wt\%Co HVOF coatings. Int J Refract Metal Hard Mater 87:105101. https://doi.org/ 10.1016/j.jirmhm.2019.105101

29. International Centre for Diffraction Data (ICDD) software Powder Diffraction File ${ }^{\mathrm{TM}}$ (PDF); https://www.icdd.com/pdfsearch/

30. Gell M, Wang J, Kumar R, Roth J, Jiang C, Jordan EH (2018) Higher temperature thermal barrier coatings with the combined use of yttrium aluminum garnet and the solution precursor plasma spray process. J Therm Spray Technol 27:543-555. https://doi.org/ 10.1007/s11666-018-0701-7

31. International organization for standardization (2005) NonDestructive Testing - Standard Test Method for Determining Residual Stresses by Neutron Diffraction, ISO/TS 21432:2005, ISO, Geneva. https://www.iso.org/standard/75266.html

32. Suzuki H, Harjo S, Abe J, Xu P, Aizawa K, Akita K (2013) Effects of gauge volume on pseudo-strain induced in strain measurement using time-of-flight neutron diffraction. Nucl Instrum Methods Phys Res, Sect A 715:28-38. https://doi.org/10.1016/j.nima.2013.03.031

33. Zhang S-Y, Godfrey E, Abbey B, Xu P, Tomota Y, Liljedahl D, Zanellato O, Fitzpatrick ME, Kelleher J, Siano S, Santisteban J, Korsunsky AM (2009) Materials structure and strain analysis using time-of-flight neutron diffraction. Proceedings of the World Congress on Engineering Vol II WCE, July 1 - 3, 2009, London, UK. http://www.iaeng.org/publication/WCE2009/WCE2009_ pp1412-1419.pdf

34. Engin-X positioner, https://www.isis.stfc.ac.uk/Pages/ENGINXPositioner.aspx

35. Von Dreele RB, Jorgensen JD, Windsor CG (1982) Rietveld refinement with spallation neutron powder diffraction data. J Appl Crystallogr 15:581-589. https://doi.org/10.1107/S0021889882012722

36. Rietveld HM (1969) A profile refinement method for nuclear and magnetic structures. J Appl Crystallogr 2:65-71. https://doi.org/ $10.1107 / \mathrm{S} 0021889869006558$

37. Simmons G, Wang H (1971) Single crystal elastic constants and calculated aggregate properties: A Handbook. MIT Press, Cambridge

38. Behnken H, Hauk V (1986) Calculation of the X-Ray elasticity constants (XEC) of the polycrystal from the elastic data of the single crystal for arbitrary crystal symmetry. Zeitschrift fuer Metallkunde/Materials Research and Advanced Techniques 77(9):620-626

39. Kesler O, Matejicek J, Sampath S, Sures S, Gnaeupel-Herold T, Brand PC, Prask HJ (1998) Measurement of residual stress in plasma-sprayed metallic, ceramic and composite coatings. Mater Sci Eng, A 257(2):215-224. https://doi.org/10.1016/S09215093(98)00860-0

40. Faisal NH, Mann L, Duncan C, Dunbar E, Clayton M, Frost M, McConnachie J, Fardan A, Ahmed R (2019) Diametral compression test method to analyse relative surface stresses in thermally sprayed coated and uncoated circular disc specimens. Surf Coat Technol 357:497-514. https://doi.org/10.1016/j.surfcoat.2018.10. 053

41. Owoseni T, Bai M, Hussain T, Faisal NH, Lee TL, Kelleher J (2018) Neutron diffraction residual stress measurements in suspension $\mathrm{HVOF}$ sprayed $\mathrm{Al}_{2} \mathrm{O}_{3}$ and $\mathrm{YSZ}$ coatings. In F. Azarmi, K. Balani, T. Eden, T. Hussain, H. Li, K. Shinoda, F.-L. Toma (eds.), Proceedings of the 2018 International thermal spray conference (ITSC 2018), 7-10 May 2018, Orlando, USA. Ohio: ASM International, pages 490-495. https://rgu-repository.worktribe.com/ OutputFile/327753

42. Ahmed R, Yu H, Stewart S, Edwards L, Santisteban JR (2007) Residual strain measurements in thermal spray cermet coatings via neutron diffraction. J Tribol 129(2):411-418. https://doi.org/ 10.1115/1.2647503

43. Ezeilo AN, Webster GA (1999) Advances in neutron diffraction for engineering residual stress measurements. Texture and Microstructures 33:151-171. https://doi.org/10.1155/TSM.33.151

44. Pirling $\mathrm{T}$ (20110 Precise analysis of near surface neutron strain imaging measurements. Procedia Engineering 10:2147-2152. https://doi.org/10.1016/j.proeng.2011.04.355

45. Venter AM, van Heerden PR, Marais D, Raaths JC (2018) MPISI: The neutron strain scanner materials probe for internal strain investigations at the SAFARI-1 research reactor. Physica B 551:417-421. https://doi.org/10.1016/j.physb.2017.12.011

46. Kirstein O, Luzin V, Garbe U (2009) The strain-scanning diffractometer Kowari. Neutron News 20(4):34-36. https://doi.org/10. 1080/10448630903241175

47. Luzin V, Vackel A, Valarezo A, Sampath S (2017) Neutron through-thickness stress measurements in coatings with high spatial resolution. Mater Sci Forum 905:165-173. https://doi.org/10. 4028/www.scientific.net/MSF.905.165

48. Oladijo OP, Luzin V, Ntsoane TP (2019) Thermally sprayed Inconel 625 coating on 304 stainless steel: a neutron diffraction stress analysis. Procedia Manufacturing 35:1234-1239. https:// doi.org/10.1016/j.promfg.2019.06.081

49. Oladijo OP, Luzin V, Maledi NB, Setswalo K, Ntsoane TP, Abe H (2020) Residual stress and wear resistance of HVOF Inconel 
625 coating on SS304 steel substrate. J Therm Spray Technol 29:1382-1395. https://doi.org/10.1007/s11666-020-01066-x

50. Smith GM, Saputo J, Luzin V, Sampath S (2020) Observation of residual stress and fatigue behavior of structurally integrated thermally sprayed nickel coatings. J Therm Spray Technol 29:12291241. https://doi.org/10.1007/s11666-020-01035-4

51. Saleh M, Luzin V, Spencer K (2014) Analysis of the residual stress and bonding mechanism in the cold spray technique using experimental and numerical methods. Surf Coat Technol 252:1528. https://doi.org/10.1016/j.surfcoat.2014.04.059

52. Luzin V, Kirstein O, Zahiri SH, Fraser D (2020) Residual stress buildup in Ti components produced by cold spray additive manufacturing (CSAM). J Therm Spray Technol 29:1498-1507. https:// doi.org/10.1007/s11666-020-01048-z

53. Kim SY, Luzin V, Sesso ML, Thornton J, Gulizia S (2020) The effect of low temperature range heat treatment on the residual stress of cold gas dynamic sprayed Inconel 718 coatings via neutron diffraction. J Therm Spray Technol 29:1477-1497. https:// doi.org/10.1007/s11666-020-01080-z

54. Gibmeier J, Back HC, Mutter M, Vollert F, Rebelo-Kornmeier J, Mücke R, Vaßen R (2018) Study of stability of microstructure and residual strain after thermal loading of plasma sprayed YSZ by through surface neutron scanning. Physica B 551:69-78. https:// doi.org/10.1016/j.physb.2017.12.014

55. Lyphout C, Nylen P, Manescu A, Pirling T (2008) Residual stresses distribution through thick HVOF sprayed inconel 718 coatings. J Therm Spray Technol 17(5-6):915-923. https://doi. org/10.1007/s11666-008-9242-9

56. Kovářík O, Haušild P, Siegl J, Pala Z, Matějíček J, Davydov V (2014) The influence of plasma sprayed multilayers of Cr2O3and Ni10wt\% Al on fatigue resistance. Surf Coat Technol 251:143150. https://doi.org/10.1016/j.surfcoat.2014.04.018

57. Gnäupel-Herold T, Matejicek J, Prask HJ (2000), Mechanical properties of plasma sprayed coatings - measured by diffraction. Proceedings of the $9^{\text {th }}$ International Metallurgical Conference Metal 2000, Ostrava, Czech Republic, paper no. 508. http:// metal2013.tanger.cz/files/proceedings/metal_00/papers/508.pdf

58. Matějíček J, Sampath S, Gnäupel-Herold T, Prask HJ (2002) Residual stress in sprayed $\mathrm{Ni}+5 \% \mathrm{Al}$ coatings determined by neutron diffraction. Appl Phys A 74:s1692-s1694. https://doi.org/10.1007/ s003390101264

59. Gnaupel-Herold T, Prask HJ, Barker J, Biancaniello FS, Jiggetts RD, Matejicek J (2006) Microstructure, mechanical properties, and adhesion in IN625 air plasma sprayed coatings. Mater Sci Eng, A 421:77-85. https://doi.org/10.1016/j.msea.2005.10.003

60. Luzin V, Prask HJ, Gnaupel-Herold T, Sampath S (2010) Use of neutron diffraction for stress measurements in thin and thick thermal sprayed coatings. International Heat Treatment and Surface Engineering 4(1):17-24. https://doi.org/10.1179/174951410X12572442577426

61. Li L, Vaidya A, Streibl T, Sampath S, Gouldstone A, Luzin V, Prask HJ (2005) Residual stress analysis of thermal sprayed molybdenum deposit. Mater Sci Forum 490-491:607 612. https://doi.org/10.4028/ www.scientific.net/MSF.490-491.607

62. Luzin V, Spencer K, Zhang M-X (2011) Residual stress and thermomechanical properties of cold spray metal coatings. Acta Mater 59:1259-1270. https://doi.org/10.1016/j.actamat.2010.10.058

63. Choi WB, Li L, Luzin V, Neiser R, Gnaupel-Herold T, Prask HJ, Sampath S, Gouldstone A (2007) Integrated characterization of cold sprayed aluminum coatings. Acta Mater 55:857-866. https:// doi.org/10.1016/j.actamat.2006.09.006

64. Luzin V, Spiridonov P, Spencer K, Gnaupel-Herold T (2020) Neutron diffraction study of macrostress and microstress in Al$\mathrm{Al}_{2} \mathrm{O}_{3}$-based corrosion protection coating obtained by cold spray (dynamic metallization). J Therm Spray Technol 29:1437-1454. https://doi.org/10.1007/s11666-020-01077-8

65. Ceretti M, Michaud M, Perrin M, A. Lodini A, (1995) Residual stress measurement in a plasma semi-transferred arc coating by neutron and x-ray diffraction. Experimental Technique 19(3):1721. https://doi.org/10.1111/j.1747-1567.1995.tb00853.x

66. Dragolici CA (2016) Experimental Methods in the Study of Neutron Scattering at Small Angles. Neutron Scattering, Waldemar Alfredo Monteiro, IntechOpen,. https://doi.org/10.5772/62184

67. Keller T, Wagner W, Ilavsky J, Margadant N, Siegmann S, Barbezat G, Pisacka J, R. Enzl R (2001) Characterization of void morphologies in thermally sprayed metallic deposits using scattering techniques. in: PSI Scientific and Technical Rep VI, ISBN/ISSN:1423-7350 91-923

68. Takajo S, Hollis KJ, Cummins DR, Tegtmeier EL, Dombrowski DE, Vogel SC (2018) Texture evolution in U-10Mo nuclear fuel foils during plasma spray coating with Zr. Quantum Beam Science 2:12. https://doi.org/10.3390/qubs2020012

69. Brokmeier HG (1997) Neutron diffraction texture analysis. Physica B 234-236:977-979. https://doi.org/10.1016/S0921-4526(96) 01230-6

70. Jakani S, Mathon M-H, Benmarouane A, Lodini A (2007) Neutron diffraction study of nano-hydroxyapatite coatings on titanium substrates. J Neutron Res 15(3):225-229. https://doi.org/10.1080/ 10238160802401211

71. Liu T, Vaudin MD, Bunn JR, Ungar T, Brewe LN (2020) Quantifying dislocation density in Al-Cu coatings produced by cold spray deposition. Acta Mater 193:115-124. https://doi.org/10. 1016/j.actamat.2020.04.040

72. Tejero-Martin D, Bai M, Mata J, Hussain T (2021) Evolution of porosity in suspension thermal sprayed YSZ thermal barrier coatings through neutron scattering and image analysis techniques. J Eur Ceram Soc 41(12):6035-6048. https://doi.org/10.1016/j.jeurceramsoc.2021.04.020

73. Woracek R, Santisteban J, Fedrigo A, Strobl M (2018) Diffraction in neutron imaging - A review. Nucl Instrum Methods Phys Res, Sect A 878:141-158. https://doi.org/10.1016/j.nima.2017.07.040

74. Venter AM, Marais D, Luzin V, Sacks N (2017) The influence of erosion wear on the residual stresses in WC-based alloy coatings. 9th International Conference on Mechanical Stress Evaluation by Neutron and Synchrotron Radiation (MECA SENS), Skukuza, South Africa, 19-21 September 2017, 75

75. Luzin V, Matějíček J, Gnäupel-Herold T (2010) Through-thickness residual stress measurement by neutron diffraction in $\mathrm{Cu}+\mathrm{W}$ plasma spray coatings. Mater Sci Forum 652:50-56. https://doi.org/10. 4028/www.scientific.net/MSF.652.50

76. Abubakar AA, Arif AFM, Al-Athel KS, Akhtar SS, Mostaghimi J (2017) Modeling residual stress development in thermal spray coatings: current status and way forward. J Therm Spray Technol 26:1115-1145. https://doi.org/10.1007/s11666-017-0590-1

77. Mathew J, Griffin J, Alamaniotis M, Kanarachos S, Fitzpatrick ME (2018) Prediction of welding residual stresses using machine learning: Comparison between neural networks and neuro-fuzzy systems. Appl Soft Comput 70:131-146. https://doi.org/10.1016/j. asoc.2018.05.017

78. Uzun F, Papadaki C, Wang Z, Korsunsky AM (2020) Neutron strain scanning for experimental validation of the artificial intelligence based eigenstrain contour method. Mech Mater 143:103316. https://doi.org/10.1016/j.mechmat.2020.103316

Publisher's Note Springer Nature remains neutral with regard to jurisdictional claims in published maps and institutional affiliations. 